van Rooijen Arnold (Orcid ID: 0000-0002-5013-2592)

Lowe Ryan, Joseph (Orcid ID: 0000-0002-7080-8406)

Rijnsdorp Dirk, Pieter (Orcid ID: 0000-0002-6409-3926)

Ghisalberti Marco (Orcid ID: 0000-0002-6690-8922)

Jacobsen Niels, Gjoel (Orcid ID: 0000-0003-2662-6282)

McCall Robert (Orcid ID: 0000-0002-9213-1539)

\title{
Wave-driven mean flow dynamics in submerged canopies
}

Arnold van Rooijen ${ }^{1,2,3,4}$, Ryan Lowe $e^{1,2,3,5,6}$, Dirk Rijnsdorp ${ }^{2,5,6}$, Marco Ghisalberti ${ }^{2,5}$, Niels G. Jacobsen ${ }^{4}$, Robert McCall ${ }^{4}$

${ }^{1}$ School of Earth Sciences, University of Western Australia, Perth, WA, Australia

${ }^{2}$ UWA Oceans Institute, University of Western Australia, Perth, WA, Australia

${ }^{3}$ ARC Centre of Excellence for Coral Reef Studies, University of Western Australia, Perth, WA, Australia

${ }^{4}$ Deltares, Delft, Netherlands

${ }^{5}$ Oceans Graduate School, University of Western Australia, Perth, WA, Australia

${ }^{6}$ Wave Energy Research Centre, University of Western Australia, Albany, WA, Australia

\begin{abstract}
The physical roughness (canopies) formed by organisms within aquatic ecosystems (e.g., seagrass, kelp, mangroves, etc.) modifies the local wave-driven hydrodynamics within coastal and estuarine regions. In wave-dominated environments, an understanding of the mean wave-driven flows generated within and above canopies is important, as it governs material transport (e.g., of nutrients, sediment and biota). However, until recently the effect of submerged canopies on wave-current interactions and the resulting mean (wave-averaged) flow dynamics has received relatively little attention. In this study, a combination of wave flume experiments and numerical modelling is used to investigate the wave-induced mean flow profiles in the presence of a submerged canopy. The measured velocities and vegetation forces were used to derive bulk drag and inertia coefficients, and to validate a non-hydrostatic 2DV wave-flow model. The numerical model results were used to conduct an in-depth analysis of the mean horizontal momentum terms responsible for driving the mean (horizontal) flow within and above the submerged canopies. We show that the mean canopy

This is the author manuscript accepted for publication and has undergone full peer review but has not been through the copyediting, typesetting, pagination and proofreading process, which may lead to differences between this version and the Version of Record. Please cite this article as doi: 10.1029/2019JC015935
\end{abstract}

This article is protected by copyright. All rights reserved. 
hydrodynamics are driven by vertical gradients in wave and turbulent Reynolds stresses, balanced by the mean canopy drag forces. The wave Reynolds stress gradient is the dominant force driving the incanopy mean flow and is directly related to the vorticity that is generated when the wave orbital motions become rotational near the canopy interface. This study provides new insight in the mechanisms responsible for wave-driven mean flows within submerged canopies and guidance for how these hydrodynamics can be predicted in coastal wave-circulation models.

\section{Plain Language Summary}

Aquatic plants that grow in estuaries and coastal oceans (such as seagrass, kelp and mangroves) have a considerable influence on water flow (currents) and on waves propagating towards the shore. However, mean flows generated by the waves interacting with aquatic vegetation (with timescales much longer than the individual wave periods) have not been comprehensively studied. This study provides a description of how submerged vegetation alters the mean wave-driven flow structure. A combination of detailed experiments conducted in a wave flume and numerical simulations are used to show that the mean flow just above the vegetation is relatively strong (up to 20 to $50 \%$ of the maximum wave velocity above the canopy), while it is considerably weaker inside the vegetation. We identified three forces that govern the mean current profile: the wave Reynolds stress gradient, the turbulent Reynolds stress gradient and the vegetation drag force. These forces are usually not accurately described in larger-scale computer simulations of coastal processes. However, reliable simulation of processes in the coastal ocean such as sediment transport and nutrient exchange requires an accurate prediction of mean flows, and thus these forces need to be properly incorporated in computer models when applied to regions with aquatic vegetation.

\section{Introduction}

Improving predictions of wave-vegetation interactions has been an increasing focus of coastal research over the past decade [e.g., Nepf, 2012], which is often motivated by the desire to quantify 
the coastal protection services provided by aquatic vegetation [e.g., Borsje et al., 2011; Tang et al., 2013]. Attenuation of wave energy due to different species of vegetation has been studied both experimentally [e.g., Fonseca and Cahalan, 1992; Möller et al., 2014] and in the field [e.g., Möller and Spencer, 2002; Paul and Amos, 2011], and analytical models have been developed based on conservation of wave energy to predict wave attenuation across a given aquatic vegetation canopy for both monochromatic [Dalrymple et al., 1984; Kobayashi et al., 1993] and irregular waves [Mendez and Losada, 2004; Suzuki et al., 2012, Jacobsen et al., 2019]. In the case of emergent vegetation and/or nonlinear waves, vegetation can contribute to a reduction in wave setup (wave-induced mean water levels) [Dean and Bender, 2006], and aquatic canopies have also been found to reduce windinduced storm surge levels by slowing storm surge advance [e.g., Sheng et al., 2012]. Although waveinduced flows are typically assumed to be depth-uniform in shallow coastal waters, the presence of submerged aquatic canopies generally results in strong vertical gradients in the wave velocities [Lowe et al., 2005; 2008; Pujol et al., 2013, Jacobsen, 2016] and localised increases in turbulence production [e.g., Neumeier and Amos, 2006].

Wave-induced mean currents within aquatic vegetation canopies have received relatively little attention to date. The vertical gradients in wave-driven mean flow within submerged canopies are thought to enhance vertical mixing of nutrients between the canopy and its surroundings [e.g., Abdolahpour et al., 2016], and on longer timescales drive local morphological changes to seabeds with canopies. Luhar et al. [2010; 2013] observed that wave-averaged mean flows were generated inside a submerged canopy formed by flexible vegetation and derived a theoretical model to quantify the magnitude of the depth-integrated wave-driven mean transport within a canopy. Their model proposes that the mean flow is induced by a nonzero wave stress, similar to streaming observed in wave boundary layers [e.g., Longuet-Higgins, 1953]. The wave stress $\left(\left\langle u_{w} w_{w}\right\rangle\right.$, where $\langle.$.$\rangle denotes$ time average) becomes nonzero when the horizontal $\left(u_{w}\right)$ and vertical $\left(w_{w}\right)$ wave velocities are no longer perfectly out of phase due to the difference in horizontal wave velocity inside and above the canopy due to canopy drag forces. The model assumes that wave energy is predominantly 
transferred from the above canopy region into the canopy through the work done by the wave-induced pressure at the top of the canopy, and that the energy transfer is balanced by the in-canopy energy dissipation dominated by work done due by the drag forces. Using linear wave theory, the governing energy balance for a canopy is then given by:

$\rho\left\langle u_{w} w_{w}\right\rangle=-\frac{k}{\omega}\left\langle\int_{0}^{h_{v}} f_{d} u d z\right\rangle$,

[Luhar et al., 2010], where $\rho$ is the water density, $k$ is the wave number, $\omega$ is the wave angular frequency, $h_{v}$ is the vegetation height, $f_{d}$ is the vegetation drag force, $u$ is the horizontal velocity, and $z$ is the vertical coordinate with origin at the seabed. Furthermore, Luhar et al. [2010] derived a timeaveraged horizontal momentum balance by assuming that the momentum transfer into the canopy is balanced by the time-averaged drag force. To derive a formulation for the mean in-canopy flow magnitude, they further assumed that: i) the drag force can readily be decomposed in a mean and wave component with drag coefficients of comparable magnitude, ii) wave energy dissipation is dominated by the wave component of the drag, iii) the mean canopy drag is dominated by the mean current in the canopy, and iv) the mean flow magnitude is constant over the height of the canopy. With these assumptions, they derived the following formulation for the depth-integrated in-canopy mean flow speed $\left(u_{m, c a n}\right)$ :

$u_{m, c a n}=\sqrt{\frac{4}{3 \pi} \frac{C_{D w}}{C_{D c}} \frac{k}{\omega} u_{w, c a n}{ }^{3}}$,

[Luhar et al., 2010], where $C_{D w}$ and $C_{D c}$ are drag coefficients associated with waves and currents respectively, and $u_{w, c a n}$ is the in-canopy wave velocity magnitude.

While the model by Luhar et al. [2010] treats the wave-induced mean flow profile inside a canopy as approximately depth-uniform, Abdolahpour et al. [2017] found relatively strong vertical gradients in the wave-induced mean velocity for submerged canopies. They also proposed a Lagrangian framework and kinematic arguments to derive an empirical relation to predict the peak value of the mean velocity near the top of a submerged canopy. Their model relies on a moving (Lagrangian) reference frame 
and thus accounts for the additional depth-varying mass flux (Stokes drift) that appears when accounting for vertical variation in the wave orbital particle motion [e.g., Phillips, 1977]. Although it is well-established that the total (Lagrangian) mass flux governs the transport of dissolved and particulate material, it can be difficult to measure Stokes drift experimentally as it requires particle tracking or use of tracers. Instead, Abdolahpour et al. [2017] validated their model using measurements from fixed (Eulerian) instruments and found a good agreement, hence suggesting the Stokes drift contribution was relatively small. However, due to the nature of their model, a clear distinction between the (Eulerian) streaming and (Lagrangian) Stokes drift influence is missing. As a consequence, a full description of the Stokes drift profile in case of submerged canopies, as well as the relative contribution to the overall wave-averaged flow dynamics is still lacking.

Other recent studies have also investigated the mean flows generated by waves interacting submerged canopies using numerical simulations [e.g., Ma et al., 2013; Chen et al., 2019]; however, these studies focused on validating the performance of specific models, and not a detailed description or explanation of the hydrodynamic mechanisms responsible for driving the flows. Although these studies have contributed to a better understanding of mean current generated through wave-canopy interaction, a comprehensive understanding of the hydrodynamic forces that governs wave-driven mean flows in submerged canopies is currently lacking.

This paper aims to increase understanding of wave-driven mean flow dynamics within submerged canopies using a combination of experimental observations and numerical modelling. In particular we aim to: i) determine the stress distributions and forces that control the mean (wave-averaged) horizontal momentum balance, ii) derive a simplified model that could replicate the vertical structure of the mean flow, iii) evaluate the contribution of Stokes drift on the mean flow dynamics, and iv) provide guidance for how these flows can be simulated within coastal scale models using wave-averaged descriptions of the hydrodynamics. 


\section{Background: the mean horizontal momentum balance}

The two-dimensional cross-shore (2DV) wave-averaged horizontal momentum equation governing the mean flow through a canopy can be derived by applying averaging operations to the Navier-Stokes equations [e.g., Nielsen, 1992]. First the velocity signal is decomposed, which for the horizontal component is defined as

$u=\langle u\rangle+\tilde{u}+u^{\prime}$

where $\langle u\rangle$ is the wave-averaged flow velocity, $\tilde{u}$ is the wave orbital velocity and $u^{\prime}$ is the turbulent velocity component. Next, the terms in the Navier-Stokes equations are time-averaged over a wave cycle (denoted by $\langle\ldots\rangle$ ) and then spatially averaged over the fluid region (i.e., neglecting the solid canopy elements):

$\frac{\partial\langle u\rangle^{2}}{\partial x}+\frac{\partial\langle u\rangle\langle w\rangle}{\partial z}+\frac{\partial\left\langle\widetilde{u}^{2}\right\rangle}{\partial x}+\frac{\partial\langle\widetilde{u} \widetilde{w}\rangle}{\partial z}+\frac{\partial\left\langle u^{\prime 2}\right\rangle}{\partial x}+\frac{\partial\left\langle u^{\prime} w^{\prime}\right\rangle}{\partial z}-v\left(\frac{\partial^{2}\langle u\rangle}{\partial x^{2}}+\frac{\partial^{2}\langle u\rangle}{\partial z^{2}}\right)+\frac{1}{\rho} \frac{\partial\langle p\rangle}{\partial x}-\frac{1}{\rho}\left\langle f_{v, x}\right\rangle=0$,

where $x$ and $z$ are the horizontal and vertical coordinates, respectively, $v$ is the kinematic viscosity, $\rho$ is the density, $p$ is the (total) pressure and $\left\langle f_{v, x}\right\rangle$ is the wave-averaged vegetation force.

The wave-averaged pressure contains a hydrostatic and non-hydrostatic component $\left(\langle p\rangle=\left\langle p_{h}\right\rangle+\right.$ $\left.\left\langle p_{n h}\right\rangle\right)$, where the hydrostatic part is given by:

$\left\langle p_{h}\right\rangle=\rho g(\langle\zeta\rangle-z)$

where $g$ is the gravitational acceleration, and $\langle\zeta\rangle$ is the mean (wave-averaged) water surface elevation. Spatial gradients in hydrostatic pressure, i.e. $\partial\left\langle p_{h}\right\rangle / \partial x$, are therefore associated with mean water level (e.g., wave setup) gradients. The non-hydrostatic pressure comprises a contribution due to the flux of vertical momentum from the vertical fluid motion and a contribution of the wave-averaged horizontal derivative of the vertically integrated shear stresses: 
$\left\langle p_{n h}\right\rangle=-\rho\left(\left\langle\widetilde{w}^{2}\right\rangle+\left\langle w^{\prime 2}\right\rangle\right)+\frac{\partial}{\partial x}\left\langle\int_{-z}^{\zeta} \rho\left(\tilde{u} \widetilde{w}+u^{\prime} w^{\prime}\right) d z\right\rangle$

which is obtained through a vertical integration of the vertical momentum equation [Svendsen, 2006]. For clarity, all terms contributing to the wave-averaged normal $\left(\sigma_{x x}\right)$ and shear $\left(\tau_{x z}\right)$ stress can be aggregated as:

$\sigma_{x x}=\rho\left(v \frac{\partial\langle u\rangle}{\partial x}-\left\langle\tilde{u}^{2}\right\rangle-\left\langle u^{\prime 2}\right\rangle\right)-\left\langle p_{n h}\right\rangle$,

$\tau_{x z}=\rho\left(v \frac{\partial\langle u\rangle}{\partial z}-\langle\tilde{u} \widetilde{w}\rangle-\left\langle u^{\prime} w^{\prime}\right\rangle\right)$,

where each of the first three terms in Eqs. (7) and (8) represent the viscous, wave-driven and turbulent contributions to the normal and shear stress, respectively. Based on these definitions, Eq. (4) can be rewritten as:

$\frac{\partial\langle u\rangle^{2}}{\partial x}+\frac{\partial\langle u\rangle\langle w\rangle}{\partial z}-\frac{1}{\rho}\left(\frac{\partial \sigma_{x x}}{\partial x}+\frac{\partial \tau_{x z}}{\partial z}-\frac{\partial\left\langle p_{h}\right\rangle}{\partial x}+\left\langle f_{d, x}\right\rangle\right)=0$.

The mean horizontal momentum balance (i.e., Eq. 4 and 9) defines the general force balance that governs the mean (wave-averaged) flow profile.

Both viscous stresses and the turbulent normal stress $\left\langle u^{\prime 2}\right\rangle$ are generally expected to be relatively small in cross-shore (2DV) coastal applications [e.g., van der Werf et al., 2017]. The wave contribution to the normal stress $\left\langle\tilde{u}^{2}\right\rangle$ is directly related to momentum associated with wave orbital motions and is often represented as the wave radiation stress through depth-integration. The wave shear stress $\langle\tilde{u} \widetilde{w}\rangle$ (also referred to as the wave Reynolds stress) is zero for irrotational waves and is therefore often neglected in coastal models. However, in many cases waves become rotational (for example, within wave boundary layers) as a result of horizontal and vertical orbital velocities being no longer $90^{\circ}$ out of phase, leading to a nonzero wave Reynolds stress [Longuet-Higgins, 1953]. Turbulence generated by vertical gradients in the mean flow generate turbulent shear stresses $\left\langle u^{\prime} w^{\prime}\right\rangle$ (also known as the turbulent Reynolds stresses).

This article is protected by copyright. All rights reserved. 
The final term, the mean horizontal vegetation force $\left\langle f_{v, x}\right\rangle$, is generally described by the (waveaveraged) Morison equation:

$\left\langle f_{v, x}\right\rangle=\left\langle f_{d, x}+f_{i, x}\right\rangle=\left\langle\frac{1}{2} \rho C_{D} d_{v} N_{v} u|u|+\rho C_{M} N_{v} A_{v} \frac{\partial u}{\partial t}\right\rangle$,

where $f_{d, x}$ and $f_{i, x}$ are the drag and inertial force respectively, $C_{D}$ is the drag coefficient, $d_{v}$ is the canopy element diameter, $N_{v}$ is the number of canopy elements per unit area, $C_{M}$ is the inertia coefficient $\left(C_{M}=1+C_{m}\right.$, where $C_{m}$ is the added mass coefficient) and $A_{v}$ is the plan surface area of a single element (for cylindrical elements, $A_{v}=\pi d_{v}^{2} / 4$ ). Although previous studies have often neglected the inertial force term, Suzuki et al. [2019] showed that its influence on wave dynamics may become significant for relatively high canopy densities.

\section{Methods}

\subsection{Wave flume experiments}

Experiments were conducted in a 35-m-long, 1.2-m-wide and 1.2-m-deep wave flume at the University of Western Australia (Figure 1). The flume was equipped with a piston-type wave maker positioned at the upstream end of the flume with a 1:10 slope at the downstream end. The slope was covered by dense polyurethane filter foam sheets, anti-fatigue rubber mats with holes and finally flat hollow concrete elements. With this approach, the slope was used as a passive wave energy absorber as waves experience progressively decreasing porosity, thereby gradually losing their energy. In a similar study in the same facility, it was found that this approach ensures wave reflections from the downstream slope are sufficiently small, with reflection coefficients $<6 \%$ [Abdolahpour et al., 2016].

A rigid submerged canopy was constructed using a staggered array of dowels (6.4 $\mathrm{mm}$ diameter) that were $30 \mathrm{~cm}$ high at a density of $\sim 3100$ units per $\mathrm{m}^{2}$, representing a relatively high-density canopy ( $\lambda_{p}=0.1$, where $\lambda_{p}$ is the canopy element plan area per unit bed area). To ensure fully developed canopy flow conditions, the length of the canopy $\left(L_{c}=2.5 \mathrm{~m}\right)$ was chosen such that $L_{c} \gg A_{\infty}$, where 
$A_{\infty}=\tilde{u}_{\max , \infty} / \omega$ is the undisturbed above-canopy wave orbital excursion length, $\tilde{u}_{\max , \infty}$ is the maximum free-stream horizontal wave orbital velocity measured just below wave trough level. Furthermore the length of the canopy was much greater than the canopy drag length scale $\left(L_{d}\right)$ defined as $L_{d} \sim 2 h_{v}\left(1-\lambda_{p}\right) / \lambda_{f}$, where $h_{v}$ is the vegetation height and $\lambda_{f}$ is the canopy element frontal area per unit bed area [e.g., Lowe et al., 2005]. Preliminary numerical modelling (presented in Section 4, e.g. Figure 5) indicated that, in all cases, edge effects were negligible at a point $1 \mathrm{~m}$ into the canopy, further validating the choice of canopy length.

The experimental program included six regular wave conditions with varying wave height $H$ and wave period $T$ (Table 1) in constant water depth $(h=0.75 \mathrm{~m})$. The Keulegan-Carpenter number $(K C=$ $\left.\tilde{u}_{\max , \infty} T d_{v}^{-1}\right)$ is a commonly used parameter in wave-canopy interaction studies, and is often used to distinguish characteristic canopy flow regimes and to provide an indication of the relative contribution of canopy drag over inertial forces [e.g., Etminan et al., 2019]. Moreover, several studies have shown that canopy drag coefficients depend on KC [e.g., Ozeren el al., 2014]. We therefore ensured that the experimental wave conditions covered a range of $K C$ (51-230, see Table 1$)$, which is considered representative for many coastal wave conditions and aquatic vegetation commonly found in nature (e.g., assuming $\tilde{u}_{\max , \infty} \sim 0.1-0.4 \mathrm{~m} / \mathrm{s}, T \sim 3-8 \mathrm{~s}$ and $d_{v} \sim 0.01 \mathrm{~m}$ resulting in $K C \sim 30-320$ ). In addition, the wave conditions cover a range of nonlinear shallow to intermediate wave conditions that are typical of areas with aquatic vegetation. Based on these conditions, the waves would generally be expected to be defined by cnoidal wave theory [Le Méhauté, 1976]; however, we note that two of the cases (R1 and R2), technically just fall within the limits of Stokes $2^{\text {nd }}$ order wave theory. Nevertheless, for convenience across all runs, the wave maker was forced using prescribed time series of the horizontal displacement of the wave paddle following the cnoidal wave generation method outlined by Goring [1979] and Cho [2003]. As a check of this approach, additional numerical model simulations of cases R1 and R2 confirmed that imposing Stokes $2^{\text {nd }}$ order wave theory had no noticeable effect on the results relative to cnoidal wave theory. 
Velocity measurements at $25 \mathrm{~Hz}$ were obtained above and within the canopy by vertically traversing a Nortek Vectrino Acoustic Doppler Velocimeter (ADV) at a location at the cross-shore mid-point of the canopy region (Figure 1). To accommodate the ADV within the canopy, a small ( $<5 \mathrm{~cm}$ diameter) area was cleared. Velocity measurements were conducted at 20 vertical positions, each for the duration of 150 wave periods. The velocity signals were decomposed by averaging the velocity signal over the total experimental duration to obtain the mean component, and subsequently ensemble averaging the velocity signal over the wave period to obtain the wave component. To assess the convergence properties (uncertainties) in the flow statistics, the flow statistics were analysed over a running interval over the experiment duration [e.g. Ting and Kirby, 1994]. For cases where the mean and turbulent flow statistics did not converge sufficiently (i.e., $>5 \%$ difference after 100 wave periods compared to statistics obtained after 150 wave periods) the data points were not included in the subsequent analysis.

To directly measure drag forces on an individual canopy element, time-series of horizontal forces exerted on a representative canopy element (aluminium dowel) was measured at $25 \mathrm{~Hz}$ using a onedimensional load cell with $2 \mathrm{~N}$ capacity (Uxcell, Hong Kong) connected to a load cell amplifier (RWST01A, SMOWO, China). The load cell itself was placed in a circular hole that was drilled inside the wave tank concrete floor. The force measurement procedure and instrument calibration are explained in detail in van Rooijen et al. [2018].

The measured time-varying depth-integrated vegetation force $\left(F_{x, m}\right)$ acting on an individual canopy element and the measured horizontal velocity were evaluated using the vertically integrated Morison equation over the height of the canopy element to estimate drag and inertia coefficients. Recent studies have shown this method provides more accurate drag coefficient estimates compared to the conventional calibration method that is based on wave height observations [e.g., Hu et al., 2014; Chen et al., 2018]. Bulk (i.e., averaged over time and canopy element height) values for the drag $\left(C_{D}\right)$ and inertia $\left(C_{M}\right)$ coefficients were obtained by applying the least-squares method (e.g. Sumer and Fredsøe [2006]). To verify whether the use of constant coefficients can explain the variation in 
vegetation force within a wave cycle (as is assumed in the numerical modelling below), the ensembleaveraged force signal was reconstructed using the measured velocity and the derived constant drag and inertia coefficients. The reconstructed force signal showed good agreement with the measured signal $\left(R^{2}>0.93\right.$ for all cases), particularly during periods of maximum force (Figure 2). However, the offshore-directed peak in the measured force is underpredicted, primarily due to a lack of an equivalent strongly-defined peak in the measured horizontal velocity when the oscillatory flow is directed offshore (and hence the reconstructed force signal). The estimated bulk drag coefficient $\left(C_{D}\right)$ decreases with increasing $R e$ and $K C$ (Figure 3; Table 1), consistent with many other studies [e.g., Ozeren et al., 2014]. The optimal inertia coefficients $\left(C_{M}\right)$, showed a relatively large scatter (mean value of 3.05, standard deviation of 2.06). This scatter was attributed to the relatively small contribution of the inertial force to the total vegetation force (Figure 2), generally $<10 \%$, leading to relatively high uncertainty in reconstructing the inertial force. In fact, additional analysis showed that the least-square error value is nearly insensitive to variations in $C_{M}$; therefore, a constant value of $C_{M}$ $=2$ was assumed throughout this study.

\subsection{Numerical model description}

\subsubsection{Governing equations}

The non-hydrostatic (RANS-based) wave-flow model SWASH (version 5.01) was used, which is effectively a direct numerical implementation of the three-dimensional Navier-Stokes equations within a terrain following framework [Zijlema et al., 2011; Smit et al., 2013; Rijnsdorp et al., 2017]. Here, the model was used in 2DV mode, for which the governing equations (momentum and continuity) are given by:

$$
\begin{aligned}
& \frac{\partial u}{\partial t}+\frac{\partial u u}{\partial x}+\frac{\partial w u}{\partial z}+\frac{1}{\rho} \frac{\partial p_{h}}{\partial x}+\frac{1}{\rho} \frac{\partial p_{n h}}{\partial x}=\frac{\partial}{\partial x}\left(v_{h} \frac{\partial u}{\partial x}\right)+\frac{\partial}{\partial z}\left(v_{v} \frac{\partial u}{\partial z}\right)+\frac{1}{\rho} f_{v, x} \\
& \frac{\partial w}{\partial t}+\frac{\partial u w}{\partial x}+\frac{\partial w w}{\partial z}+\frac{1}{\rho} \frac{\partial p_{n h}}{\partial z}=\frac{\partial}{\partial z}\left(v_{v} \frac{\partial w}{\partial z}\right)+\frac{\partial}{\partial x}\left(v_{h} \frac{\partial w}{\partial x}\right) \\
& \frac{\partial u}{\partial x}+\frac{\partial w}{\partial z}=0
\end{aligned}
$$


$\frac{\partial \zeta}{\partial t}+\frac{\partial \int_{-h}^{\zeta} u}{\partial x}=0$

where $f_{v, x}$ is the (instantaneous) vegetation force, and $\zeta$ is the water surface elevation. Within the canopy, the horizontal vegetation force $\left(f_{v, x}\right)$ is computed using the Morison equation based on the known vegetation canopy properties and the local instantaneous horizontal velocity [Suzuki et al., 2019]:

$f_{v, x}=\frac{1}{2} \rho C_{d} d_{v} N_{v} u|u|+\rho C_{M} N_{v} A_{v} \frac{\partial u}{\partial t}$.

The canopy porosity is accounted for by using a pore velocity inside the canopy, i.e. the spatially averaged velocity takes into account the space occupied by the canopy [see Suzuki et al., 2019, for details].

In SWASH, the turbulent stresses (i.e., first and second terms on the RHS of Eqs. (11) and (12)) are approximated using the eddy viscosity concept, where $v_{v}$ and $v_{h}$ are the vertical and horizontal eddy viscosity, respectively. The vertical eddy viscosity $\left(v_{v}\right)$ is obtained using a turbulence closure $(k-\varepsilon)$ model extended with vegetation effects. The $k-\varepsilon$ equations (which include vegetation effects) are given by:

$\frac{\partial k}{\partial t}+\frac{\partial k w}{\partial z}=\frac{\partial}{\partial z}\left(\frac{v_{v}}{\sigma_{k}} \frac{\partial k}{\partial z}\right)+P_{k}+C_{f k} P_{v}-\varepsilon$,

$\frac{\partial \varepsilon}{\partial t}+\frac{\partial \varepsilon w}{\partial z}=\frac{\partial}{\partial z}\left(\frac{v_{v}}{\sigma_{\varepsilon}} \frac{\partial \varepsilon}{\partial x}\right)+C_{1 \varepsilon} \frac{\varepsilon}{k}\left(P_{k}+C_{f \varepsilon} P_{v}\right)-C_{2 \varepsilon} \frac{\varepsilon^{2}}{k}$,

where the vertical eddy viscosity is computed as:

$v_{v}=C_{\mu} \frac{k^{2}}{\varepsilon}$

The turbulence production consists of a component due to shear $\left(P_{k}\right)$ and due to vegetation, which only considers production by drag forces $\left(P_{v}\right)$ :

$P_{k}=v_{v}\left(\frac{\partial u}{\partial z}\right)^{2}$ 
$P_{v}=f_{d} u=\frac{1}{2} C_{d} d_{v} N_{v}|u|^{3}$.

The model contains a number of universal constants (i.e., $C_{\mu}=0.09, C_{1 \varepsilon}=1.44, C_{2 \varepsilon}=1.92, \sigma_{k}=1$ and $\left.\sigma_{\varepsilon}=1.3\right)$ which were derived by Launder and Spalding [1974]. The coefficients related specifically to vegetation drag $\left(C_{f k}\right.$ and $\left.C_{f \varepsilon}\right)$, can be set by the user and are discussed in the next section. To allow for horizontal turbulent mixing, the Prandtl mixing length approximation is used to estimate the horizontal eddy viscosity $v_{h}$, with the mixing length taken as equal to the wave height [as per Zijlema et al., 2011].

In this study, the traditional cell-centred arrangement for the non-hydrostatic pressure variables combined with the central difference scheme for the vertical non-hydrostatic pressure gradient was used. In the case of fine vertical resolutions (>10 layers) as used in this work, this numerical scheme allows the wave characteristics to be captured (e.g., wave dispersion and wave nonlinearity) with sufficient accuracy [e.g., Smit et al., 2013] and is preferable over the Keller-Box method [e.g. Zijlema et al., 2011] due to its computational efficiency.

\subsubsection{Model schematization}

A horizontally uniform computational grid resolution of $0.1 \mathrm{~m}$ was used along the model domain $(32 \mathrm{~m})$ to ensure a minimum of 50 grid points per wavelength were resolved when simulating each experimental case. The water column was resolved using 40 vertical computational layers with a varying resolution of $0.06 \mathrm{~m}$ (near bottom) to $0.001 \mathrm{~m}$ (near the top of the canopy interface). To minimise interpolation inaccuracies during the post-processing of the model results when computing the contributions to the mean momentum balance, the vertical profile was resolved using a combination of layers with fixed (i.e., z-layers) and variable thicknesses (i.e., sigma-layers). A total of $36 z$-layers were located between the bottom $(z=0 \mathrm{~m})$ and $z=0.5 \mathrm{~m}$ (i.e., $0.2 \mathrm{~m}$ above the canopy), with the remaining part of the water column was resolved using 4 equidistant sigma-layers. Initial grid sensitivity testing showed that this vertical resolution was required for accurate results, while a higher resolution did not improve the results substantially. 
The canopy was specified within the SWASH vegetation module by replicating the canopy height $\left(h_{v}=\right.$ $0.3 \mathrm{~m})$, canopy element diameter $\left(d_{v}\right)$ and density $\left(N_{v}\right)$ that were used in the experiments. The drag force coefficients were obtained from the experimental measurements, whereas for the inertia coefficients we assumed default values $\left(C_{M}=2\right)$ given the small relative magnitude of inertial forces that made quantifying $C_{M}$ in the experiments difficult (see Section 3.1). The turbulence model requires two empirical coefficients $\left(C_{f k}\right.$ and $\left.C_{f \varepsilon}\right)$ related to the energy transfer from mean and oscillatory flow into turbulent energy due to vegetation drag. Here, we used the values as recommended by López and García $[1997 ; 1998]$ for vegetated flows (i.e. $C_{f k}=1$ and $C_{f \varepsilon}=1.33$ ), values that were also used by Ma et al. [2013] who applied (similar) non-hydrostatic wave-flow model to study turbulence and wave damping induced by submerged canopies.

\subsubsection{Boundary conditions and modelling procedure}

To reproduce the waves within the experiment, second-order cnoidal wave theory was used to force the model wave maker, consistent with the method used for the physical wave maker. At the bottom boundary the logarithmic wall law option was used assuming a smooth bed. The model was subsequently run for each experimental case (Table 1) for a duration equal to 150 wave periods (equal to the measurement duration). To allow the hydrodynamics to spin up to a steady state, only the last 100 wave periods were used when analysing both measurements and model results.

\subsubsection{Lagrangian particle tracking model}

To study the Eulerian and Stokes drift contribution to the Lagrangian transport, we used highresolution output from SWASH in combination with an offline coupled particle tracking model (described in Appendix A) to simulate the Lagrangian particle trajectories. Over 100 particles were released across the water column with highest density around the top of the canopy for a fixed (midcanopy) horizontal location. The time step was set to $0.01 \mathrm{~s}$ to obtain accurate simulation of particle trajectories. The wave-averaged (horizontal) Lagrangian velocity profile was obtained by computing the vector displacement of each particle over a wave cycle. The resulting mean velocity profile was mildly sensitive to the moment of particle release with respect to the wave phase. Hence, to provide a 
robust measure of the mean transport, each particle tracking simulation was repeated 50 times (with release times distributed uniformly over the wave cycle), with results from the 50 simulations subsequently averaged.

\section{Results}

\subsection{Mean, wave and turbulent velocities}

The measured and modelled velocities were decomposed into mean, wave and turbulence contributions, and normalised by $\tilde{u}_{r m s, \infty}$, i.e., the measured root-mean-square wave velocity at a reference elevation $z=2 h_{v}$ above the canopy (Table 1 ). The vertical structure of the unsteady wave component of the horizontal velocity (represented by the root-mean-square value $\tilde{u}_{r m s}$ ) modelled with SWASH agrees well with the observations (Figure 4, top panels). The wave orbital velocities are substantially attenuated within the canopy (typically by $40-60 \%$ ), whereas there is a localised peak in velocity located just above the canopy characteristic for oscillatory boundary layer flow. The measured mean velocity profile $\langle u\rangle$ is generally well-captured by the model (Figure 4 , bottom panels) with a region of enhanced onshore flow near the top of the canopy that is also consistent with previous experimental studies [e.g., Abdolahpour et al., 2017]. Within the canopy, mean flow velocities are nearly zero (R1-R3) to slightly negative (offshore directed, R4-R6), while far above the canopy an offshore directed mean current is found in all cases. Near the top of the canopy, the mean flow velocity is relatively high in direction of wave propagation, reaching values of up to 20 to $50 \%$ of $\tilde{u}_{r m s, \infty}$.

The spatial distribution of the cross-shore mean velocity field across the canopy is further explored using the model results for case R3 (see Figure 5). Both offshore and onshore of the canopy region, the mean flow was directed opposite to the direction of wave propagation and is mostly uniform over the depth. At the leading edge of the canopy, a counter-clockwise eddy forms (centred $0.2 \mathrm{~m}$ offshore the canopy) that causes an upward flow at the leading edge. At about $0.5 \mathrm{~m}$ upstream of the canopy, 
the mean flow changes direction and increases in strength, while far above the canopy the flow is still directed against the direction of wave propagation. Within $1 \mathrm{~m}$ downstream of the canopy leading edge, the relatively strong mean flow on top of the canopy is formed which persists until the trailing edge of the canopy. At this trailing edge, a clockwise rotating eddy forms with opposite direction and with a centre slightly below the top of the canopy.

Finally, we can also compare the measured in-canopy depth-integrated mean velocities with the values predicted by the analytical model given by Eq. (2) [Luhar et al., 2010] (see Table 1). For all cases the in-canopy mean flow velocity is overestimated, while for case R2-R5 the depth-integrated mean flow is even offshore directed. This may be due to the generation of a return current (undertow) in the wave flume, which was found negligible in the experiments by Luhar et al. [2010]. Another explanation may be the fact that the analytical model does not have any dependencies on canopy properties, such as canopy density. The current experiments use a relatively high canopy density, which may be violating some of the assumptions in the derivation of Eq. (2). Finally, it may be the case that the height of the flexible canopy $(6 \mathrm{~cm}$ to $10 \mathrm{~cm})$ in Luhar et al. [2010] is simply small compared to the thickness of the streaming layer, which is why the relatively strong onshore-directed mean flow induced by wave-canopy interaction can penetrate a larger fraction of the canopy. In the present study, the canopy was high at $30 \mathrm{~cm}$.

\subsection{Lagrangian transport and the effect of Stokes drift}

Based on the particle motions computed by the particle tracking routine, the mean Lagrangian velocity profile $\langle u\rangle^{L}$ follows a similar pattern to the fixed-reference (Eulerian) mean velocity profile $\langle u\rangle^{E}$ (Figure 6). This indicates the Langrangian transport is dominated by the Eulerian contribution over most of the water column, whereas the Stokes drift effect $\langle u\rangle^{S}$ (obtained by subtracting the Eulerian from the Lagrangian mean velocity) is relatively small. Locally just below the top of the canopy, the Stokes drift velocity is seen to have a considerable contribution to the total transport velocity. The Stokes drift velocity generally shows a slightly increasing magnitude from bottom to the water surface, except for the area near the top of the canopy. Just below the top of the canopy there is a peak in the Stokes 
drift, which is consistent throughout all six cases. For reference, the maximum mean flow velocity as predicted by Abdolahpour et al. [2017] is plotted as well (Figure 6). Their model is able to accurately predict the maximum mean velocity for case $\mathrm{R} 1, \mathrm{R} 2, \mathrm{R} 3$ and $\mathrm{R} 5$, while it substantially overestimates the flow for case R4 and R6. For both cases, the return current is relatively strong within the canopy compared to the other cases (where the return flow is relatively strong above the canopy), and it appears that this current may be responsible for diminishing the streaming just above the canopy.

Given that the total (Lagrangian) mass flux is dominated by the Eulerian contribution (the latter is the direct output from both measurements and the wave model), the subsequent analysis focuses on investigating the Eulerian mean flow dynamics in further detail.

\subsection{Contributions to the wave-averaged horizontal momentum balance}

To investigate the mechanisms responsible for the wave-induced mean (Eulerian) velocity profile, we evaluated all terms in the momentum balance per Eqs. (4) and (9). Since our experimental measurements do not contain observations at different horizontal locations across the canopy, for this analysis we focus on using the validated model results to interrogate the spatial distribution of the individual momentum terms. In the following, case R3 is used to highlight results representative for all cases.

All terms in Eq. (4) were computed and plotted over depth at a location $(x=1.75 \mathrm{~m})$ far enough from the leading edge to avoid edge effects (Figure 7). For all cases, the shear stress gradient $\partial \tau_{x z} / \partial z$ is the dominant term, and is comprised of both wave and turbulent Reynolds stress gradients $\left(-\partial\langle\tilde{u} \widetilde{w}\rangle / \partial z\right.$ and $-\partial\left\langle u^{\prime} w^{\prime}\right\rangle / \partial z$, respectively). The two dominant force contributions (i.e. the Reynolds stress gradients) are balanced by the wave-averaged drag forces within the canopy while balancing each other above the canopy. Note that in this case one term (wave normal stress gradient, $\partial\left\langle\tilde{u}^{2}\right\rangle / \partial x$, grey line in Figure 7) appears non-negligible further above the canopy. The relatively large wave normal stress gradient is, however, not representative for the other runs as will be shown in the following. While the residual mean momentum in this analysis is considerably smaller than each of the 
dominant momentum terms, it is not exactly zero, which is likely due to a combination of numerical inaccuracies and vertical interpolation in the post-processing.

Figure 8 reveals the spatial variability in the wave-averaged normal and shear stress gradients, $\partial \sigma_{x x} / \partial x$ and $\partial \tau_{x z} / \partial z$, respectively, over the domain. Offshore of the canopy, the normal stress gradient is relatively small and depth uniform (Figure 8A); whereas, a relatively strong negative normal stress gradient develops just offshore of the leading edge of the canopy, which appears to drive the counter-clockwise eddy that forms locally (Figure 5). Within the canopy, the normal stress gradient is relatively large over only a small portion $(<1 \mathrm{~m})$ near the leading edge, which is due to relatively strong wave energy attenuation that is generally known to follow an exponential decay [e.g., Kobayashi et al., 1993]. While the normal shear stress gradient also appears relatively strong near the trailing edge, within the interior region of the canopy its magnitude is small. Above the canopy the normal stress gradient is relatively small and may drive the return flow over the canopy (Figure 5).

The shear stress gradient term is, apart from a small region near the leading edge of the canopy, only dominant near the top of the canopy (Figure 8B), and is responsible for the strong mean current just above the top of the canopy (Figure 5). In this study, we are particularly interested in fully developed mean canopy hydrodynamics (i.e., no leading or trailing edge effects, e.g. $1 \mathrm{~m}<x<2 \mathrm{~m}$ ) for which Figure 8 suggests that the normal stress gradients are relatively small compared to the shear stress gradients. However, the simulations are based on experiments with a relatively short canopy $(2.5 \mathrm{~m})$ carried out in a closed wave flume which may result in return flows that are less likely in open coast field settings. To verify these results for more realistic (unconfined) field conditions, we also conducted three additional simulations based on case R3 but with a total numerical flume length of $100 \mathrm{~m}$, a canopy length of $20 \mathrm{~m}$ (about 2.5 times the wave length) and an absorbing wave boundary downstream of the canopy, which shows a very similar spatial distribution of normal and shear stress gradients (see Supporting Information), and thus confirms the dominance of the shear stress gradient near the top of the canopy. 
After dividing the water column into an area within the canopy and above the canopy, the momentum terms were further integrated over the depth of each region and normalized with the total mean drag force providing a clear summary of the governing forces in the wave-averaged momentum balance for all six cases (Figure 9). Although there is some variation among the cases, generally it is found that the drag within the canopy is almost completely balanced by the shear stress gradient (consisting of a wave and a turbulent Reynolds stress gradient). Above the canopy the wave Reynolds stress gradient is equal and opposite to the turbulent Reynolds stress gradient. Other momentum terms also become non-negligible in specific cases; for example, the normal stress gradient (case R2 and R3), and both the hydrostatic (case R1 and R3) and non-hydrostatic pressure gradients (case R1). However, no consistent pattern was found in the response of these terms, and could also change sign depending on the case (Figure 9). The hydrostatic pressure term (most substantial in case R1 and R3) is related to a gradient in the mean water level along the length of the canopy (i.e. any gradients in wave setup).

\section{Discussion}

The results provide insight into the wave-averaged force balances that are responsible for determining the mean wave-driven flow inside and just above the canopy generated through interactions with the canopy. The analysis revealed that the force balances within and just above the canopy were dominated by three terms: the wave and turbulent Reynolds stress gradients and the mean canopy drag forces, thus equivalent to:

$\frac{\partial\langle\widetilde{u} \widetilde{w}\rangle}{\partial z}+\frac{\partial\left\langle u^{\prime} w^{\prime}\right\rangle}{\partial z}-\frac{1}{\rho}\left\langle f_{v, x}\right\rangle=0$.

This balance is analogous to the balance found for unidirectional submerged canopy flows [e.g., Ghisalberti, 2010], but with the inclusion of a wave Reynolds stress gradient term. In the following sections the physical significance of each force term will be discussed in more detail. Although the wave Reynolds stress gradient was found to be the dominant driving force, the turbulent Reynolds stress gradient was often still important and hence incorporated. 


\subsection{The wave Reynolds stress: relationship to vertical wave motions and vorticity at the canopy interface}

The wave Reynolds stress is the dominant driver of the mean flows generated within the submerged canopies. Luhar et al. [2010] derived their expression for depth-integrated in-canopy mean flow by assuming that the turbulent stress is negligible and that linear wave theory is valid. By combining expressions for $\langle\tilde{u} \widetilde{w}\rangle$ from energy and force balances (i.e., assuming that the wave Reynolds stress gradient balances drag), their method does not require explicit computation of the wave Reynolds stress gradient in to determine the mean velocity. This method only provides a prediction for the depth-integrated mean velocity (and wave Reynolds stress), and does not predict the vertical profile, and hence the flow maxima observed near the top of the canopy. In order to be able to predict the vertical variation in wave-averaged canopy flow, i.e. $\partial\langle u\rangle / \partial z$, the vertical distribution of the mean wave Reynolds stress $(\partial\langle\tilde{u} \widetilde{w}\rangle / \partial z)$ is needed.

The appearance of the wave Reynolds stress gradient as dominant term can be shown to be directly related to the vorticity generated when wave orbital motions become rotational once they interact with the discontinuity in drag at the canopy interface. Rivero and Arcilla [1995] derived a general analytical expression for the vertical distribution of $\langle\tilde{u} \widetilde{w}\rangle$ in relation to the (wave) normal stresses $\left(\left\langle\tilde{u}^{2}\right\rangle\right.$ and $\left.\left\langle\widetilde{w}^{2}\right\rangle\right)$ and the vorticity of the oscillatory flow $(\widetilde{\omega})$ :

$\frac{\partial\langle\widetilde{u} \widetilde{w}\rangle}{\partial z}=\langle\widetilde{w} \widetilde{\omega}\rangle-\frac{1}{2} \frac{\partial\left(\left\langle\widetilde{u}^{2}\right\rangle-\left\langle\widetilde{w}^{2}\right\rangle\right)}{\partial x}$,

where the oscillatory vorticity is given by:

$\widetilde{\omega}=\frac{\partial \widetilde{u}}{\partial z}-\frac{\partial \widetilde{w}}{\partial x}$

Several studies have used the relationship in Eq. (22) to predict mean velocity profiles (e.g. due to undertow [e.g., Garcez Faria et al., 2000; Guannel and Özkan-Haller, 2014]). However, expressions are typically derived by assuming that linear wave theory is valid and that waves are irrotational, hence $\widetilde{\omega} \approx 0$, which allows for the vorticity term (first term on RHS of Eq. (22)) to be neglected. The

This article is protected by copyright. All rights reserved. 
remaining term (i.e., the second term on the RHS of Eq. (22)) can then readily be obtained from any wave theory (e.g., linear wave theory).

Since, by definition, the wave-averaged values $\langle\tilde{u}\rangle$ and $\langle\widetilde{w}\rangle$ equal zero, the wave-averaged value of the oscillatory vorticity $\langle\widetilde{\omega}\rangle$ also equals zero. However, as seen in Eq. (22), it is the quantity $\langle\widetilde{w} \widetilde{\omega}\rangle$ that is directly related to the vertical distribution of the wave Reynolds stress [Rivero and Arcilla, 1995]. The vorticity term determines the wave Reynolds stress gradient near the top of the canopy, while the contribution of the wave normal stress gradient (second term on RHS of Eq. (22)) is negligible (Figure $10 \mathrm{~A}-\mathrm{C}$, representative for all cases). So in order to further study the contributions to the vorticity effect near the top of a submerged canopy, Eq. (22) can be rewritten as:

$\frac{\partial\langle\widetilde{u} \widetilde{w}\rangle}{\partial z} \approx\langle\widetilde{w} \widetilde{\omega}\rangle=\left\langle\widetilde{w} \frac{\partial \widetilde{u}}{\partial z}-\widetilde{w} \frac{\partial \widetilde{w}}{\partial x}\right\rangle=\left\langle\widetilde{w} \frac{\partial \tilde{u}}{\partial z}\right\rangle-\left\langle\widetilde{w} \frac{\partial \widetilde{w}}{\partial x}\right\rangle$.

As may be expected for canopy flows with strong vertical gradients in the horizontal orbital velocity (Figure 4), the first term on the RHS of Eq. (24) dominates the vorticity effect on the wave shear stress (Figure 10D,E). Hence, this shows that the vertical gradient in wave stress can be approximated by:

$\frac{\partial\langle\widetilde{u} \widetilde{w}\rangle}{\partial z} \approx\langle\widetilde{w} \widetilde{\omega}\rangle \approx\left\langle\widetilde{w} \frac{\partial \widetilde{u}}{\partial z}\right\rangle$

where the horizontal and vertical orbital velocity components may be obtained from 1DV models [e.g., Zeller et al., 2015; Jacobsen, 2016], which can therefore provide a means to predict wave-driven mean flow profiles within coupled (phase-averaged) wave ocean circulation models (see section 5.4). Note that the term $\langle\widetilde{w} \partial \widetilde{w} / \partial x\rangle$ may be more important than shown in the present case for larger nondimensional water depths $(k h)$ and away from the top of the canopy.

\subsection{Turbulent Reynolds stress}

For unidirectional flows, the vertical gradient in turbulent Reynolds stress is generated by the shear at the top of the canopy and is known to drive the in-canopy flow together with the horizontal pressure gradient [Nepf and Vivoni, 2000]. In this present study of wave-driven flows, we found that the 
turbulent Reynolds stress was consistently smaller than the wave Reynolds stress, but is still an important force that contributes to the structure of the wave-averaged mean flow profile. However, we also acknowledge that these results may depend to some degree on the $k-\varepsilon$ turbulence closure model in SWASH that relies on two empirical vegetation coefficients. Prior studies have generally used two different sets of coefficients to account for vegetation effects in $k-\varepsilon$ models [see discussion by Defina and Bixio, 2005]. Some authors use the values recommenced by Lopez and Garcia [1997, 1998] where $C_{f k}=1$ and $C_{f \varepsilon}=1.33$ (as in this study), others apply the values as proposed by Shimizu and Tsujimoto [1994] $\left(C_{f k}=0.07\right.$ and $\left.C_{f \varepsilon}=0.16\right)$. To study the sensitivity of the model results to these coefficients, simulations were repeated for two additional cases: (i) with the alternative coefficients suggested by Shimizu and Tsujimoto [1994], and (ii) without the turbulence model. The resulting mean and RMS velocity in case of the alternative set of coefficients are nearly identical to the results obtained when not using a turbulence model at all (see example for case R3 in Figure 11). The above-canopy peak in the mean velocity using the original turbulence settings is smaller and overall the turbulent Reynolds stress appears to diminish the peak velocity (Figure 11A). The peak in RMS velocity just above the canopy is also smaller compared to the peak obtained for the alternative turbulence settings or no turbulence model at all (Figure 11B). In all cases, the mean momentum balance is dominated by the wave Reynolds stress gradient (Figure 11C) balanced by the canopy drag forces (Figure 11E), while the turbulent Reynolds stress gradient is smaller (Figure 11D). With the original settings a smoother transition of both wave and turbulent Reynolds stresses from the location with the largest shear (top of the canopy) to the regions inside and above the canopy is obtained, resulting in smoother velocity profiles. Although these results do not affect the overall conclusions of the present work, further research on the turbulent Reynolds stress in wave-driven canopy flows would be helpful in order to reduce uncertainty in estimating its relative contribution on canopy flow dynamics. Recent studies on wave-canopy interaction using more detailed wave models that are less reliant on turbulence parameterizations [e.g., Chakrabarti et al., 2016; Etminan et al., 2019] have the potential to partly fill this gap, particularly in combination with ever-increasing computational capabilities. 


\subsection{Wave-averaged canopy drag force}

In wave-only conditions, the wave-averaged drag force in a submerged canopy is zero for linear (sinusoidal) waves and is consequently often ignored in phase-averaged wave modelling studies. However, in practice a nonzero wave-averaged drag force appears when mean flows are present or if the waves are nonlinear [Dean and Bender, 2006]. Moreover, an additional, and potentially considerable wave-current interaction term emerges from the full decomposition of the mean drag force and subsequent wave-averaging (accounting for the absolute velocity functionality in the total drag formulation):

$$
\begin{aligned}
\left\langle f_{d, x}\right\rangle & =\beta\langle u|u|\rangle, \\
& =\beta\langle(\langle u\rangle+\tilde{u})|\langle u\rangle+\tilde{u}|\rangle, \\
& =\beta\left(\left\langle\frac{u}{|u|}\right\rangle\langle u\rangle|\langle u\rangle|+\left\langle\frac{u}{|u|} \tilde{u}|\tilde{u}|\right\rangle+2\left\langle\frac{u}{|u|}\langle u\rangle \tilde{u}\right\rangle\right),
\end{aligned}
$$

where $\beta=\frac{1}{2} \rho C_{D} d_{v} N_{v}$. In previous studies on drag due to combined wave-current flows interacting with canopies, studies have typically assumed that the interaction term (i.e., the third term on the RHS of Eq. (26)) is negligible and the total drag can be readily decomposed into mean and oscillatory drag components [e.g., Zhou and Graham, 2000; van Rooijen et al., 2016]. In the present work, it is found that when not accounting for the interaction components, the total simulated mean drag force may be overestimated in the lower part of the canopy and underestimated near the top of the canopy for some of the cases investigated (Figure 12A, B). The relative contribution of each component on the total (depth-integrated) $\operatorname{drag}\left(F_{d}^{\text {rel }}=F_{d}^{\text {component }} / F_{d}^{\langle u|u|\rangle}\right)$ varies among the different cases. For instance, for case R1, R2 and R3 the mean drag is dominated by the wave and the interaction terms while the component associated with mean flow is negligible (as highlighted in Figure $12 \mathrm{C}$ for R3). However, for the remaining cases, both the (depth-integrated) mean and interaction terms are negligible contributions to the total depth-integrated canopy drag compared to the wave-related term (see example for R4 in Figure 12D). These results indicate that the interaction terms may not always be 
negligible, or may even be dominant, and cannot be ignored when studying wave-averaged canopy flow dynamics.

\subsection{Implications for coupled phase-averaged wave ocean circulation models}

Although phase-resolving wave-flow models are increasingly being used on coastal scales, including 3D and 2DV models with vertical resolution, they are still computationally too expensive for many practical broad-scale coastal applications. Phase-averaged wave models coupled to ocean circulation models are therefore still widely used to predict coastal flows, and such models are also the foundation for most coastal sediment transport applications (e.g., Delft3D, ROMS-COAWST). In this study, we identified three force terms that govern the vertical distribution of the momentum balances that determines the mean wave-driven mean flow inside and above the canopy. Although our study was limited to regular waves, this streaming effect is also expected to occur for irregular waves, which is consistent with observations reported in the field [Luhar et al., 2013]. While the development of analytical formulations for embedding wave-driven canopy flow interactions within coupled wavecirculation models is beyond the scope of the present study, the results provide preliminary insight into how these hydrodynamics can be incorporated in large-scale, practical engineering models. This would allow for a better description of the mean transport processes within a range of coastal ecosystems that form submerged canopies, which in turn would help to provide more accurate prediction of processes as sediment transport and nutrient exchange.

\section{Conclusions}

In this study, the wave-averaged mean flow dynamics within submerged canopies were investigated using a combination of wave flume experiments and numerical modelling based on a multi-layered 2DV non-hydrostatic (phase-resolving) wave model. Through direct measurement of flow velocities and forces exerted on a canopy element, drag coefficients were derived for each experimental case and used as input for the numerical model. The model was able to simulate the complex flow dynamics when waves interact with submerged canopies, including the vertical distribution of the 
mean velocity profile, which contained a peak mean velocity just above the top of the canopy. A particle tracking routine was applied to evaluate the Lagrangian transport processes, which revealed that the relative contribution of Stokes drift is relatively small compared to the Eulerian flux over most of the water column, yet locally important adjacent to the top of the canopy. A wave-averaged momentum budget analysis demonstrated that the wave-driven mean flows inside and just above the canopy were driven by a combination of vertical gradients in the wave $\langle\tilde{u} \widetilde{w}\rangle$ and turbulent $\left\langle u^{\prime} w^{\prime}\right\rangle$ Reynolds stresses, balanced by wave-averaged canopy drag forces. In order to model realistic approximations of the mean velocity profile in and above submerged canopies, these terms need to be accounted for in coastal models. However, due to the complexity involved in each individual term, analytical derivations are required to derive practical parameterizations that can be implemented in large scale (phase-averaged) coastal models.

\section{APPENDIX A: LAGRANGIAN PARTICLE TRACKING MODEL}

A MATLAB-based particle tracking model was developed to compute the Lagrangian flow within and above a submerged vegetation canopy (see Section 4.2). The model is based on a $4^{\text {th }}$ order RungeKutta advection scheme, as commonly applied in ocean particle tracking models [e.g., North et al., 2006]. Particles were released instantaneously at $>100$ vertical locations for one fixed horizontal position at mid-canopy, and the model was run for a full wave cycle with a time step of $0.01 \mathrm{~s}$. To provide a robust measure of the mean transport the simulations were repeated 50 times resulting in an ensemble of simulations differing $T / 50$ in particle release time. The resulting particle vector displacements and Lagrangian velocities were then averaged over all ensemble simulations to obtain a representative Lagrangian mean velocity profile. Some additional details regarding the interpolation scheme and advection sub-model are provided in the following sections.

The instantaneous horizontal and vertical (Eulerian) velocity computed by SWASH is used to calculate the particle trajectory after release at a certain $\left(x_{p}, z_{p}\right)$-position within the model domain. The velocities are interpolated to the initial particle locations using a cubic spline method. Since the

This article is protected by copyright. All rights reserved. 
particle tracking time step $(0.01 \mathrm{~s})$ is smaller than the SWASH output time step (0.1 s), an interpolation in time is required as well. For this, the velocities are estimated at the particle location for the 5 consecutive SWASH output time points nearest to the current particle tracking time point. Next a cubic spline interpolation is applied to interpolate the velocities from the 5 (SWASH) time points to the time of particle motion.

To calculate the movement of particles due to advection, a $4^{\text {th }}$ order Runge-Kutta scheme in space and time is used. It uses velocities calculated by SWASH at previous and future times to provide a robust estimate of the particle motion throughout a wave cycle. The location of a particle at a certain time step is determined by the location at the previous time step plus the weighted average of four increments:

$x_{p, n}=x_{p, n-1}+\frac{1}{6}\left(k_{u, 1}+2 k_{u, 2}+2 k_{u, 3}+k_{u, 4}\right)$,

$z_{p, n}=z_{p, n-1}+\frac{1}{6}\left(k_{w, 1}+2 k_{w, 2}+2 k_{w, 3}+k_{w, 4}\right)$.

The increments are based on the estimated slope between the previous and the current location, e.g.:

$k_{u, 1}=\Delta t \cdot u_{p, n-1}\left(x_{p, n-1}, z_{p, n-1}\right)$

$k_{u, 2}=\Delta t \cdot u_{p, n-1}\left(x_{p, n-1}+\frac{k_{u, 1}}{2}, z_{p, n-1}+\frac{k_{w, 1}}{2}\right)$

$k_{u, 3}=\Delta t \cdot u_{p, n-1}\left(x_{p, n-1}+\frac{k_{u, 2}}{2}, z_{p, n-1}+\frac{k_{w, 2}}{2}\right)$

$k_{u, 4}=\Delta t \cdot u_{p, n-1}\left(x_{p, n-1}+\frac{k_{u, 3}}{2}, z_{p, n-1}+\frac{k_{w, 3}}{2}\right)$

and equally so for the vertical component $\left(k_{w, 1}-k_{w, 4}\right)$. Here, $n$ is defined as the current particle time point.

This article is protected by copyright. All rights reserved. 


\section{References}

Abdolahpour, M., Ghisalberti, M., Lavery, P., and McMahon, K. (2016). Vertical mixing in coastal canopies. Limnology and Oceanography, 62(1), 26-42, doi: 10.1002/Ino.10368

Abdolahpour, M., Hambleton, M., and Ghisalberti, M. (2017). The wave-driven current in coastal canopies. Journal of Geophysical Research: Oceans, 122(5), 3660-3674, doi: 10.1002/2016JC012446.

Borsje, B. W., van Wesenbeeck, B. K., Dekker, F., Paalvast, P., Bouma, T. J., van Katwijk, M. M., and de Vries, M. B. (2011). How ecological engineering can serve in coastal protection. Ecological Engineering, 37(2), 113-122, doi: 10.1016/j.ecoleng.2010.11.027.

Chakrabarti, A., Chen, Q., Smith, H. D., and Liu, D. (2016). Large eddy simulation of unidirectional and wave flows through vegetation. Journal of Engineering Mechanics, 142(8), 04016048, doi: 10.1061/(ASCE)EM.1943-7889.0001087.

Chen, H., Ni, Y., Li, Y., Liu, F., Ou, S., Su, M., Yisheng, P., Hu, Z., Uijttewaal, W. and Suzuki, T. (2018). Deriving vegetation drag coefficients in combined wave-current flows by calibration and direct measurement methods. Advances in water resources, 122, 217-227. Doi: 10.1016/j.advwatres.2018.10.008

Chen, H., Liu, X., and Zou, Q. P. (2019). Wave-driven flow induced by suspended and submerged canopies. Advances in water resources, 123, 160-172. doi: 10.1016/j.advwatres.2018.11.009

Cho, Y. S. (2003). A note on estimation of the Jacobian elliptic parameter in cnoidal wave theory. Ocean engineering, 30(15), 1915-1922, doi: 10.1016/S0029-8018(03)00040-4.

Dalrymple, R.A., Kirby, J.T. and Hwang, P.A. (1984). Wave diffraction due to areas of energy dissipation. Journal of Waterway, Port, Coastal and Ocean Engineering, 110(1), 67-79, doi: 10.1061/(ASCE)0733-950X(1984)110:1(67).

Dean, R. G., and Bender, C. J. (2006). Static wave setup with emphasis on damping effects by vegetation and bottom friction. Coastal engineering, 53(2-3), 149-156, doi: 10.1016/j.coastaleng.2005.10.005.

Defina, A., and Bixio, A. C. (2005). Mean flow and turbulence in vegetated open channel flow. Water Resources Research, 41(7), doi: 10.1029/2004WR003475.

Etminan, V., Lowe, R. J., and Ghisalberti, M. (2019). Canopy resistance on oscillatory flows, Coastal Engineering (In Press), doi: 10.1016/j.coastaleng.2019.04.014.

Fonseca, M. S., and Cahalan, J. A. (1992). A preliminary evaluation of wave attenuation by four species of seagrass. Estuarine, Coastal and Shelf Science, 35(6), 565-576, doi: 10.1016/S0272-7714(05)80039-3.

Garcez Faria, A. F., Thornton, E. B., Lippmann, T. C., and Stanton, T. P. (2000). Undertow over a barred beach. Journal of Geophysical Research: Oceans, 105(C7), 16999-17010, doi: 10.1029/2000JC900084.

Ghisalberti, M. (2010). The three-dimensionality of obstructed shear flows, Environmental Fluid Mechanics, 10 (3), 329-343, doi: 10.1007/s10652-009-9161-4. 
Goring, D. G. (1979). Tsunamis-the propagation of long waves onto a shelf. PhD thesis, California Institute of Technology. Report No. KH-R-38.

Guannel, G., and Özkan-Haller, H. T. (2014). Formulation of the undertow using linear wave theory. Physics of Fluids, 26(5), 056604, doi: 10.1063/1.4872160.

Hu, Z., Suzuki, T., Zitman, T., Uittewaal, W., and Stive, M. (2014). Laboratory study on wave dissipation by vegetation in combined current-wave flow. Coastal Engineering, 88, 131-142, doi: 10.1016/j.coastaleng.2014.02.009.

Jacobsen, N. G. (2016). Wave-averaged properties in a submerged canopy: Energy density, energy flux, radiation stresses and Stokes drift. Coastal Engineering, 117, 57-69, doi: 10.1016/j.coastaleng.2016.07.009.

Jacobsen, N.G., McFall, B. C. and Van der A, D. A. (2019). A frequency distributed dissipation model for canopies, Coastal Engineering, 150, 135-146, doi: 10.1016/j.coastaleng.2019.04.007.

Keulegan, G. H. and Carpenter, L. H. (1958). Forces on cylinders and plates in an oscillating fluid. J. Research of the National Bureau of Standards, 2857, 423-440.

Kobayashi, N., Raichle, A. W., and Asano, T. (1993). Wave attenuation by vegetation. Journal of waterway, port, coastal, and ocean engineering, 119(1), 30-48, doi: 10.1061/(ASCE)0733950X(1993)119:1(30).

Launder, B. E., and Spalding, D. B. (1983). The numerical computation of turbulent flows. Numerical Prediction of Flow, Heat Transfer, Turbulence and Combustion, pp. 96-116, doi: 10.1016/B978-0-08-030937-8.50016-7.

Le Méhauté, B. (1976). An introduction to hydrodynamics and water waves. Springer Study Edition, Springer, Berlin, Heidelberg, doi: 10.1007/978-3-642-85567-2_15.

Longuet-Higgins, M. S. (1953). Mass transport in water waves. Philosophical Transactions of the Royal Society of London. Series A, Mathematical and Physical Sciences, 245(903), 535-581, doi: 10.1098/rsta.1953.0006.

López, F., and García, M. (1997). Open-Channel Flow Through Simulated Vegetation: Turbulence Modeling and Sediment Transport. Technical Report WRP-CP-10, University of Illinois at Urbana-Champaign, USA.

López, F., and García, M. (1998). Open-channel flow through simulated vegetation: Suspended sediment transport modeling. Water resources research, 34(9), 2341-2352, doi: 10.1029/98WR01922.

Lowe, R. J., Koseff, J. R., and Monismith, S. G. (2005). Oscillatory flow through submerged canopies: 1. Velocity structure. Journal of Geophysical Research: Oceans, 110(C10), doi: 10.1029/2004JC002788.

Lowe, R. J., Shavit, U., Falter, J. L., Koseff, J. R., and Monismith, S. G. (2008). Modeling flow in coral communities with and without waves: A synthesis of porous media and canopy flow approaches. Limnology and Oceanography, 53(6), 2668-2680, doi: 10.4319/lo.2008.53.6.2668. 
Luhar, M., Coutu, S., Infantes, E., Fox, S., and Nepf, H. (2010). Wave-induced velocities inside a model seagrass bed. Journal of Geophysical Research: Oceans, 115(C12), doi: 10.1029/2010JC006345.

Luhar, M., Infantes, E., Orfila, A., Terrados, J., and Nepf, H. M. (2013). Field observations of waveinduced streaming through a submerged seagrass (Posidonia oceanica) meadow. Journal of Geophysical Research: Oceans, 118(4), 1955-1968, doi: 10.1002/jgrc.20162.

Ma, G., Kirby, J. T., Su, S. F., Figlus, J., and Shi, F. (2013). Numerical study of turbulence and wave damping induced by vegetation canopies. Coastal Engineering, 80, 68-78, doi: 10.1016/j.coastaleng.2013.05.007.

Mendez, F. J., and Losada, I. J. (2004). An empirical model to estimate the propagation of random breaking and nonbreaking waves over vegetation fields. Coastal Engineering, 51(2), 103-118, doi: 10.1016/j.coastaleng.2003.11.003.

Möller, I., and Spencer, T. (2002). Wave dissipation over macro-tidal saltmarshes: Effects of marsh edge typology and vegetation change. Journal of Coastal Research, 36(sp1), 506-521, doi: 10.2112/1551-5036-36.sp1.506.

Möller, I., Kudella, M., Rupprecht, F., Spencer, T., Paul, M., Van Wesenbeeck, B. K., Wolters, G., Jensen, K. Bouma, T. J., Lange, M. M. and Schimmels, S. (2014). Wave attenuation over coastal salt marshes under storm surge conditions. Nature Geoscience, 7(10), 727, doi: 10.1038/ngeo2251.

Nepf, H. M. (2012). Flow and transport in regions with aquatic vegetation. Annual review of fluid mechanics, 44, 123-142, doi: 10.1146/annurev-fluid-120710-101048.

Nepf, H. M., and Vivoni, E. R. (2000). Flow structure in depth-limited, vegetated flow. Journal of Geophysical Research: Oceans, 105(C12), 28547-28557, doi: 10.1029/2000JC900145.

Neumeier, U. R. S., and Amos, C. L. (2006). The influence of vegetation on turbulence and flow velocities in European salt-marshes. Sedimentology, 53(2), 259-277, doi: 10.1111/j.13653091.2006.00772.x.

Nielsen, P. (1992). Coastal bottom boundary layers and sediment transport, Advanced Series on Ocean Engineering (Vol. 4). World Scientific, Singapore.

North, E. W., R. R. Hood, S.-Y. Chao, and L. P. Sanford. (2006). Using a random displacement model to simulate turbulent particle motion in a baroclinic frontal zone: a new implementation scheme and model performance tests. Journal of Marine Systems, 60: 365-380, doi: 10.1016/j.jmarsys.2005.08.003.

Ozeren, Y., Wren, D. G., and Wu, W. (2014). Experimental investigation of wave attenuation through model and live vegetation. Journal of Waterway, Port, Coastal, and Ocean Engineering, 140(5), 04014019, doi: 10.1061/(ASCE)WW.1943-5460.0000251.

Ozeren, Y., Wren, D., and Wu, W. (2017). Wave setup on vegetated beach: laboratory experiments. Proceedings of the International Conference on Coastal Engineering 2016, 1(35), 4, doi: 10.9753/icce.v35.currents.4. 
Paul, M., and Amos, C. L. (2011). Spatial and seasonal variation in wave attenuation over Zostera noltii. Journal of Geophysical Research: Oceans, 116(C8), doi: 10.1029/2010JC006797.

Phillips, O. M. (1977). The dynamics of the upper ocean. Second edition. Cambridge University Press, University of Cambridge, United Kingdom.

Pujol, D., Serra, T., Colomer, J., and Casamitjana, X. (2013). Flow structure in canopy models dominated by progressive waves. Journal of hydrology, 486, 281-292, doi: 10.1016/j.jhydrol.2013.01.024.

Rijnsdorp, D. P., Smit, P. B., Zijlema, M., and Reniers, A. J. (2017). Efficient non-hydrostatic modelling of 3D wave-induced currents using a subgrid approach. Ocean Modelling, 116, 118-133, doi: 10.1016/j.ocemod.2017.06.012.

Rivero, F. J., and Arcilla, A. S. (1995). On the vertical distribution of < ũ $\mathbf{x}>$. Coastal Engineering, 25(34), 137-152, doi: 10.1016/0378-3839(95)00008-Y.

Roelvink, J. A. and Reniers, A. J. H. M. (2011). A guide to modelling coastal morphology, Advances in Coastal and Ocean Engineering (Vol. 12). World Scientific, Singapore.

Sheng, Y. P., Lapetina, A., and Ma, G. (2012). The reduction of storm surge by vegetation canopies: Three-dimensional simulations. Geophysical Research Letters, 39(20), doi: 10.1029/2012GL053577.

Shimizu, Y., and Tsujimoto, T. (1994). Numerical analysis of turbulent open-channel flow over a vegetation layer using a $\mathrm{k}-\varepsilon$ turbulence model. Journal of Hydroscience and Hydraulic Engineering, JSCE, 11(2), 57-67.

Smit, P., Zijlema, M., and Stelling, G. (2013). Depth-induced wave breaking in a non-hydrostatic, near-shore wave model. Coastal Engineering, 76, 1-16, doi: 10.1016/j.coastaleng.2013.01.008.

Stelling, G., and Zijlema, M. (2003). An accurate and efficient finite-difference algorithm for nonhydrostatic free-surface flow with application to wave propagation. International Journal for Numerical Methods in Fluids, 43(1), 1-23, doi: 10.1002/fld.595.

Sumer, B.M., and Fredsøe, J. (2006), Hydrodynamics around cylindrical structures (revised edition), Advanced Series on Ocean Engineering, Vol. 26, World Scientific, Singapore. ISBN 981-270039-0.

Suzuki, T., Zijlema, M., Burger, B., Meijer, M. C., and Narayan, S. (2012). Wave dissipation by vegetation with layer schematization in SWAN. Coastal Engineering, 59(1), 64-71, doi: 10.1016/j.coastaleng.2011.07.006.

Suzuki, T., Hu, Z., Kumada, K., Phan, L. K., and Zijlema, M. (2019). Non-hydrostatic modeling of drag, inertia and porous effects in wave propagation over dense vegetation fields. Coastal Engineering, 149, 49-64, doi: 10.1016/j.coastaleng.2019.03.011.

Svendsen, I. A. (2006). Introduction to nearshore hydrodynamics. Advanced Series on Ocean Engineering, Vol., 24, World Scientific, Singapore. 
Tang, J., Causon, D., Mingham, C., and Qian, L. (2013). Numerical study of vegetation damping effects on solitary wave run-up using the nonlinear shallow water equations. Coastal Engineering, 75, 21-28, doi: 10.1016/j.coastaleng.2013.01.002.

Ting, F. C. K., and Kirby, J. T. (1994). Observation of undertow and turbulence in a laboratory surf zone. Coastal Engineering, 24, 5l-80, doi: 10.1016/0378-3839(94)90026-4.

van der Werf, J., Ribberink, J., Kranenburg, W., Neessen, K., and Boers, M. (2017). Contributions to the wave-mean momentum balance in the surf zone. Coastal engineering, 121, 212-220, doi: 10.1016/j.coastaleng.2016.12.007.

van Rooijen, A. A., McCall, R. T., Van Thiel de Vries, J. S. M., Van Dongeren, A. R., Reniers, A. J. H. M., and Roelvink, J. A. (2016). Modeling the effect of wave-vegetation interaction on wave setup. Journal of Geophysical Research: Oceans, 121(6), 4341-4359, doi: 10.1002/2015JC011392.

van Rooijen, A., Lowe, R., Ghisalberti, M., Conde-Frias, M., and Tan, L. (2018). Predicting currentinduced drag in emergent and submerged aquatic vegetation canopies. Frontiers in Marine Science, 5, 449, doi: 10.3389/fmars.2018.00449.

Zeller, R. B., Zarama, F. J., Weitzman, J. S., and Koseff, J. R. (2015). A simple and practical model for combined wave-current canopy flows. Journal of Fluid Mechanics, 767, 842-880, doi: 10.1017/jfm.2015.59.

Zhou, C. Y., and Graham, J. M. R. (2000). A numerical study of cylinders in waves and currents. Journal of Fluids and Structures, 14(3), 403-428, doi: 10.1006/jfls.1999.0276.

Zijlema, M., Stelling, G., and Smit, P. (2011). SWASH: An operational public domain code for simulating wave fields and rapidly varied flows in coastal waters. Coastal Engineering, 58(10), 992-1012, doi: 10.1016/j.coastaleng.2011.05.01

\section{Acknowledgments}

This research was undertaken by AvR as part of a Ph.D. at The University of Western Australia who was funded by a University Postgraduate Award for International Students (UPAIS) and an Australian Government Research Training Program (RTP) Scholarship. AvR and RL acknowledge funding provided by the ARC Centre of Excellence for Coral Reef Studies. The authors would like to thank Mr Carlin Bowyer, Ms Dianne King, and Mr Brad Rose for assistance in the laboratory, Dr Guido Wager for providing support with the force sensor, and Dr Jana Orszaghova and Dr Betsy Seiffert for providing advice on wave generation in the wave flume. We would like to thank the Associate Editor (Dr Ryan Mulligan) and the constructive feedback from Dr Tomo Suzuki and an anonymous reviewer that helped improve this manuscript. The experimental data and numerical model input files can be accessed via https://research-repository.uwa.edu.au/en/datasets/laboratory-experiments-andnumerical-modelling-of-wave-driven-mea. 


\section{Tables}

Table 1: Experimental conditions for all cases: offshore wave height $(H)$ upstream of the canopy, wave period $(T)$, Reynolds number $(R e)$, Keulegan-Carpenter number $(K C)$, drag coefficient $\left(C_{d}\right)$ derived from combined force sensor and velocity measurements (Section 3.1), the above-canopy root-mean-square velocity $\left(\tilde{u}_{r m s, \infty}\right)$, the measured depth-integrated in-canopy mean flow velocity $\left(\langle u\rangle_{c a n}\right)$, and the depth-integrated in-canopy mean flow velocity as predicted by Luhar et al. [2010] $\left(\langle u\rangle_{\text {can,Luhar }}\right)$.

\begin{tabular}{|l|l|l|l|l|l|l|l|l|}
\hline $\begin{array}{l}\text { Run } \\
\text { ID }\end{array}$ & $H[\mathrm{~m}]$ & $T[\mathrm{~s}]$ & $R e$ & $K C$ & $C_{d}$ & $\begin{array}{l}\tilde{u}_{r m s, \infty} \\
{\left[\mathrm{m} \mathrm{s}^{-1}\right]}\end{array}$ & $\begin{array}{l}\langle u\rangle_{\text {can }} \\
{\left[\mathrm{m} \mathrm{s}^{-1}\right]}\end{array}$ & $\begin{array}{l}\langle u\rangle_{\text {can,Luhar }} \\
{\left[\mathrm{m} \mathrm{s}^{-1}\right]}\end{array}$ \\
\hline R1 & 0.14 & 2 & 1043 & 51 & 2.00 & 0.14 & 0.003 & 0.019 \\
\hline R2 & 0.10 & 3 & 1009 & 74 & 1.84 & 0.13 & -0.007 & 0.014 \\
\hline R3 & 0.21 & 3 & 1845 & 135 & 1.14 & 0.28 & -0.005 & 0.025 \\
\hline R4 & 0.20 & 4 & 1727 & 169 & 0.95 & 0.29 & -0.008 & 0.017 \\
\hline R5 & 0.09 & 5 & 1182 & 144 & 1.22 & 0.13 & -0.014 & 0.008 \\
\hline R6 & 0.21 & 5 & 1887 & 230 & 0.85 & 0.26 & -0.017 & 0.014 \\
\hline
\end{tabular}




\section{Figure captions}

Figure 1: Schematic diagram of experimental setup (with vertical scale exaggerated) with location of Nortek Vectrino Acoustic Doppler Velocimeter (ADV) and load cell (LC).

Figure 2: Instantaneous vegetation force (ensemble-averaged by wave phase) from direct force measurement $\left(F_{m}\right)$ and reconstructed using velocity measurements based on the optimal force coefficients $C_{D}$ and $C_{M}\left(F_{r}=F_{r, d}+F_{r, i}\right)$, including the separate contributions of the drag $\left(F_{r, d}\right)$ and inertial $\left(F_{r, i}\right)$ forces, shown for case R3.

Figure 3: Drag coefficients $\left(C_{D}\right)$ derived from drag force and velocity measurement as function of $(\mathrm{A})$ Reynolds number, and (B) Keulegan-Carpenter number.

Figure 4: Dimensionless root-mean-square (top) and wave-averaged velocity (bottom) for cases R1 R6 obtained from measurements (markers) and model (line).

Figure 5: Modelled dimensionless wave-averaged horizontal velocity magnitude (color) and mean flow direction (vectors) around the canopy (dashed line) as function of $x$ (where the leading edge of the canopy is at $x=0 \mathrm{~m}$ ) and $\mathrm{z}$ (focused on the lower portion of the water column, i.e. below the wave troughs) for case R3.

Figure 6: Simulated Eulerian (black), Lagrangian (orange) and Stokes drift (blue) mean velocity profiles for all cases R1-R6, as well as the values for the peak mean velocity as predicted by Abdolahpour et al. [2017, triangles].

Figure 7: Wave-averaged mid-canopy $(x=1.3 \mathrm{~m})$ momentum budget terms over depth for (case R3). All terms not listed in the legend are plotted in grey. 
Figure 8: Modelled dimensionless wave-averaged (A) normal stress gradient $\partial \sigma_{x x} / \partial x$, and (B) shear stress gradient $\partial \tau_{x z} / \partial z$ around the canopy (dashed line) as function of $x$ (where the leading edge of the canopy is at $x=0 \mathrm{~m}$ ) and $z$ (focused on the lower portion of the water column, i.e. below the wave troughs) for case R3.

Figure 9: Dimensionless wave-averaged mid-canopy momentum budget terms (following Eq. (1) and normalising with the wave-averaged depth-integrated canopy drag force) integrated over different regions of the water column (A) above canopy $\left(z>h_{v}\right)$ and (B) inside canopy $\left(z<h_{v}\right)$.

Figure 10: Relationship between the (A) wave Reynolds stress and vorticity around the canopy (dashed line) as function of $x$ and $z$ for case R3, with (B, C) the two components on the RHS of Eq. (19) and (D, E) the two components on the RHS of Eq. (21).

Figure 11: Sensitivity of (A) dimensionless mean velocity, (B) dimensionless RMS velocity, (C) wave Reynolds stress gradient, (D) turbulent Reynolds stress gradient, and (E) mean canopy drag force for varying turbulence model settings for case R3.

Figure 12: Modelled (A, B) total mean drag force (black line) and contributions as function of depth, and (C, D) relative depth-integrated mean drag force contributions $\left(F_{d}^{\text {rel }}=F_{d}^{\text {component }} / F_{d}{ }^{<u|u|>}\right)$ for case (A, C) R3 and (B, D) R4. 


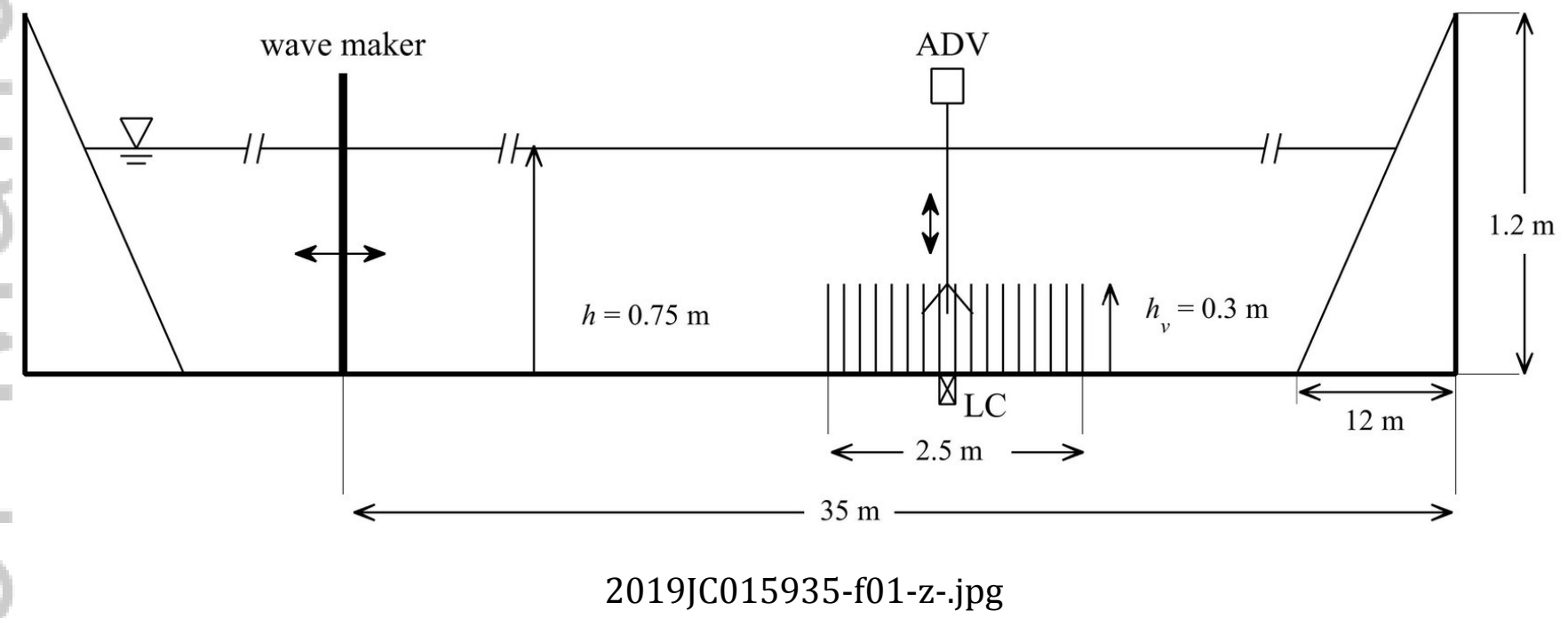

This article is protected by copyright. All rights reserved. 


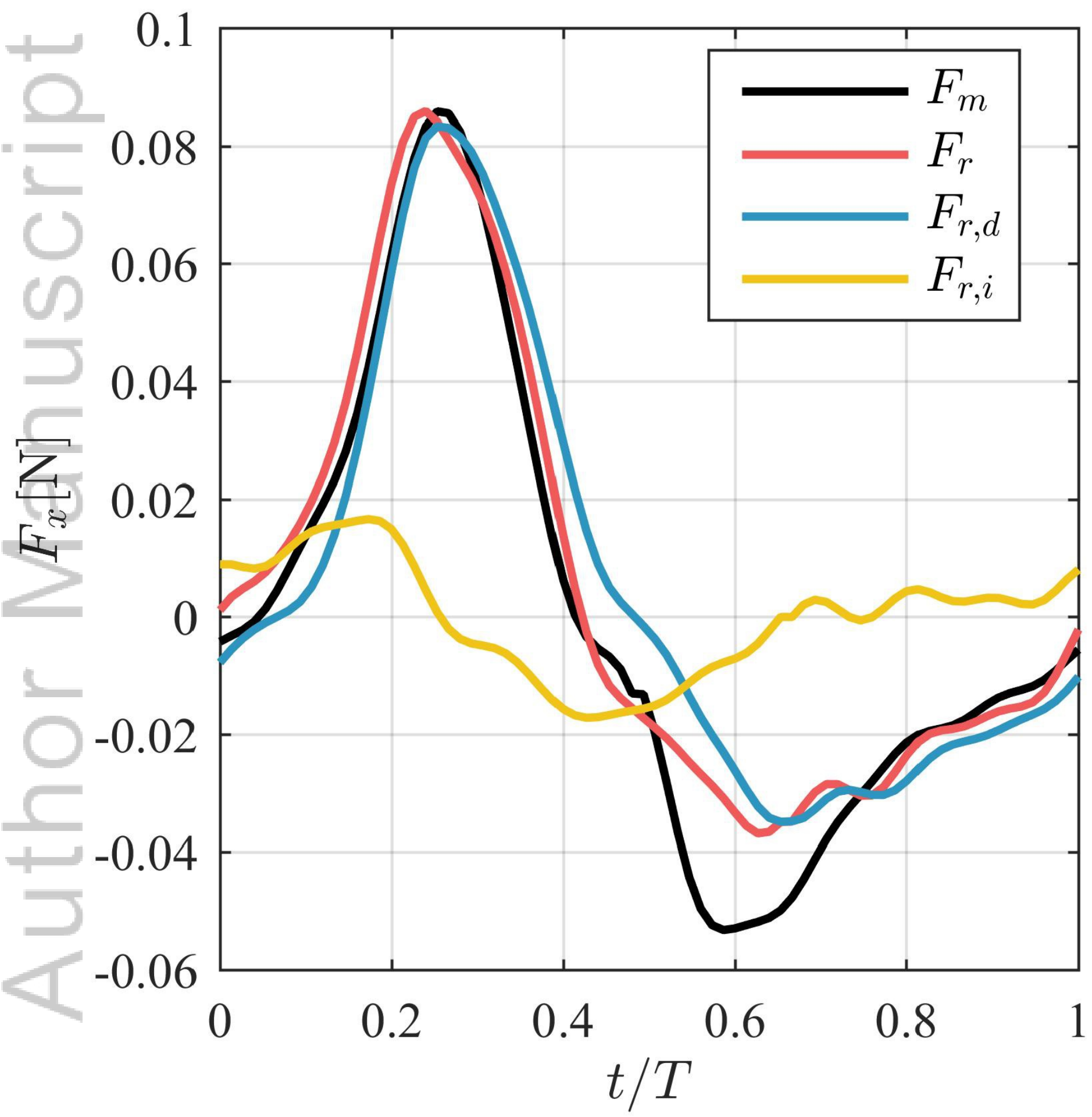

2019JC015935-f02-z-.jpg

This article is protected by copyright. All rights reserved. 

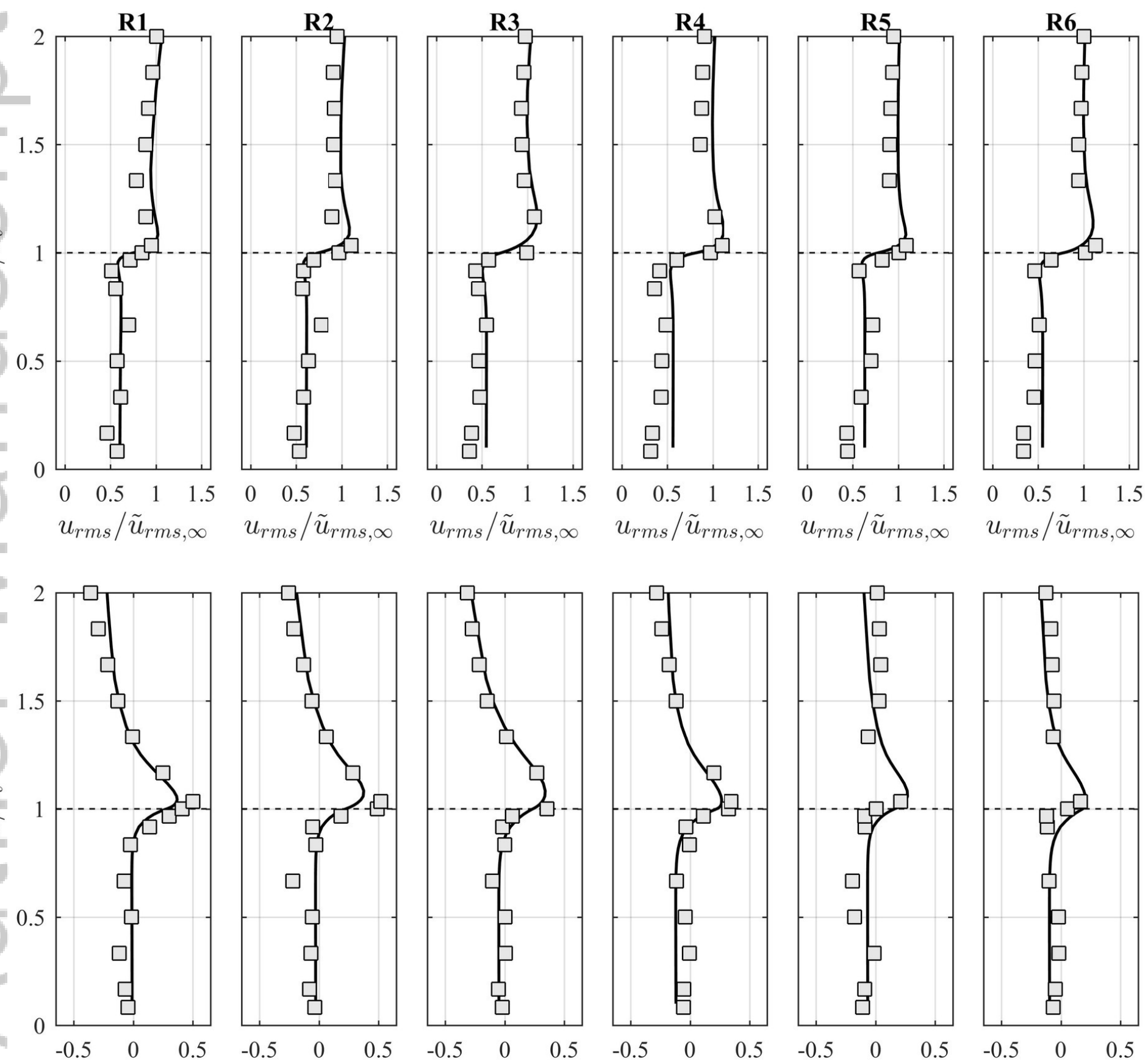

$\langle u\rangle / \tilde{u}_{r m s, \infty}$

$\langle u\rangle / \tilde{u}_{r m s, \infty}$

$\langle u\rangle / \tilde{u}_{r m s, \infty}$

$\langle u\rangle / \tilde{u}_{r m s, \infty}$

$\langle u\rangle / \tilde{u}_{r m s, \infty}$

$\langle u\rangle / \tilde{u}_{r m s, \infty}$

2019JC015935-f03-z-.jpg 

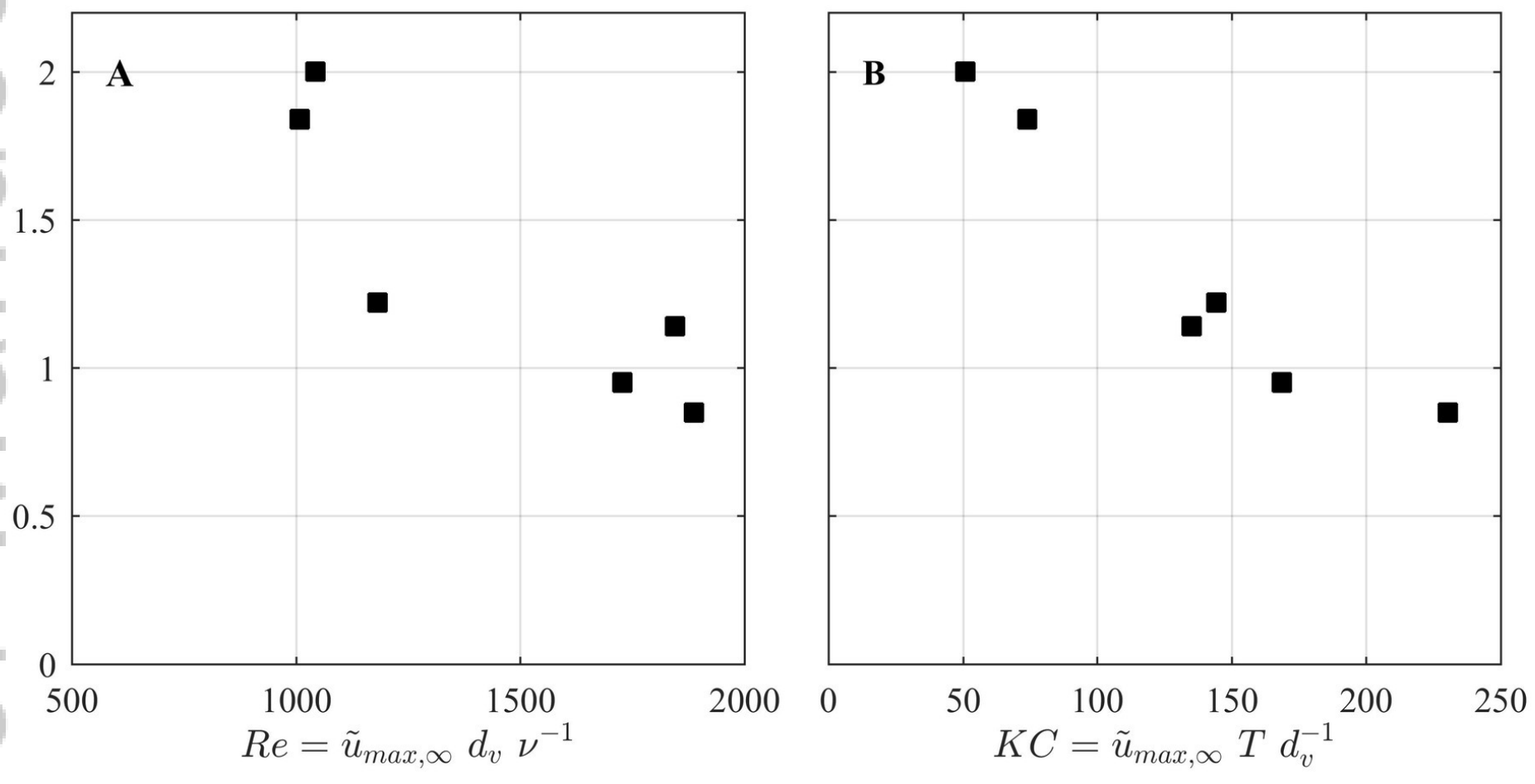

2019JC015935-f04-z-.jpg

This article is protected by copyright. All rights reserved. 

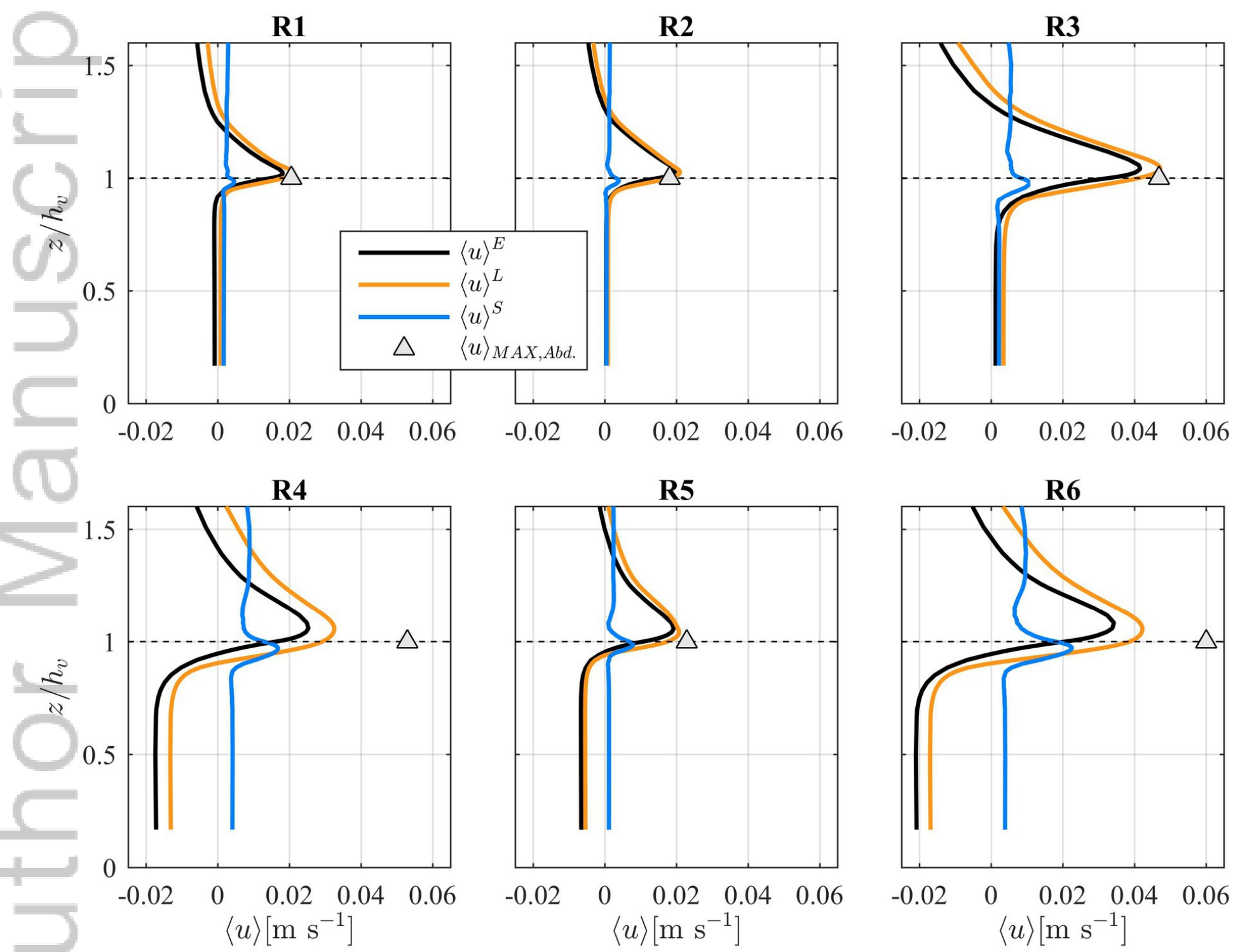

2019JC015935-f05-Z-.jpg 


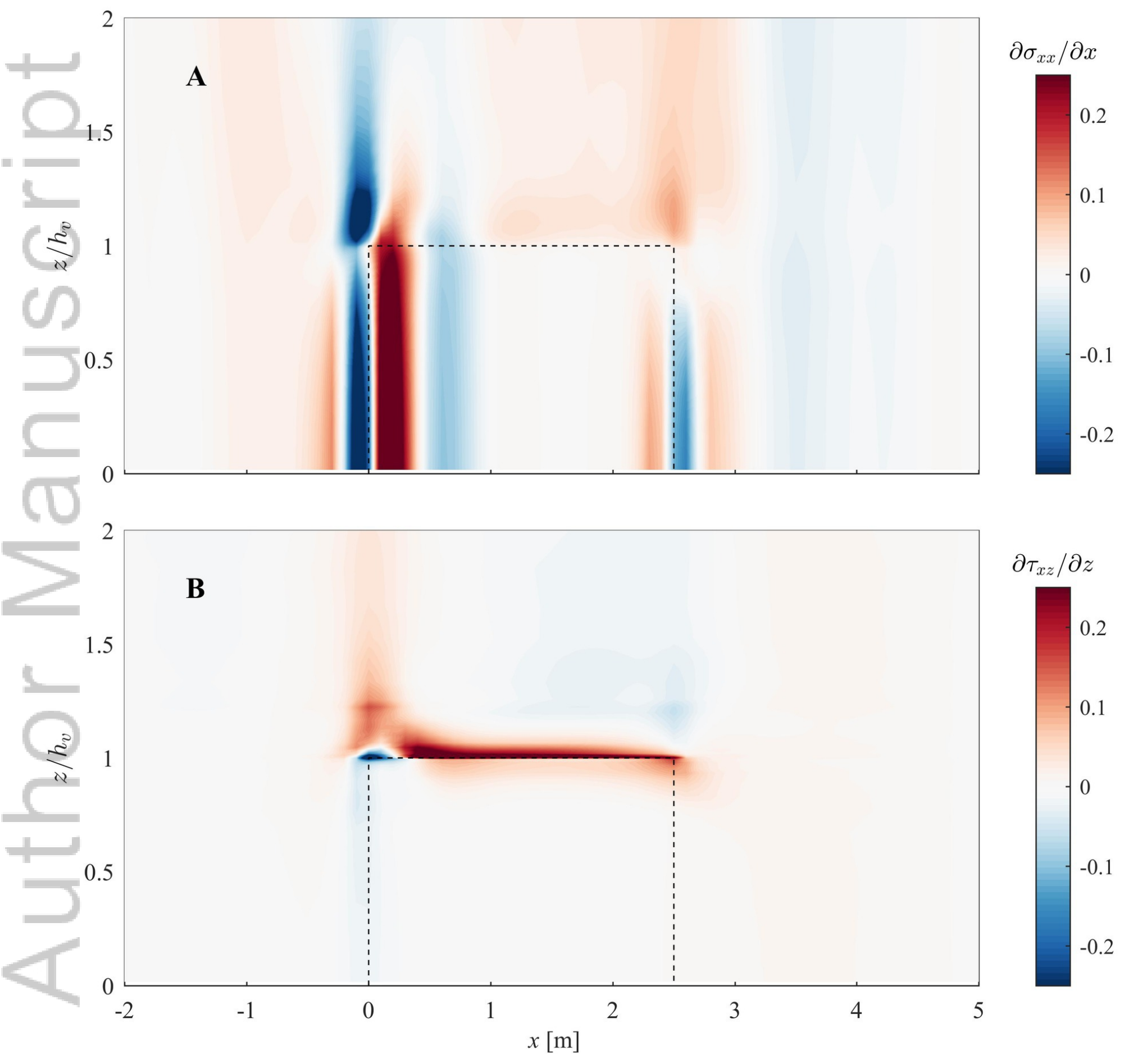

2019JC015935-f06-z-.jpg 

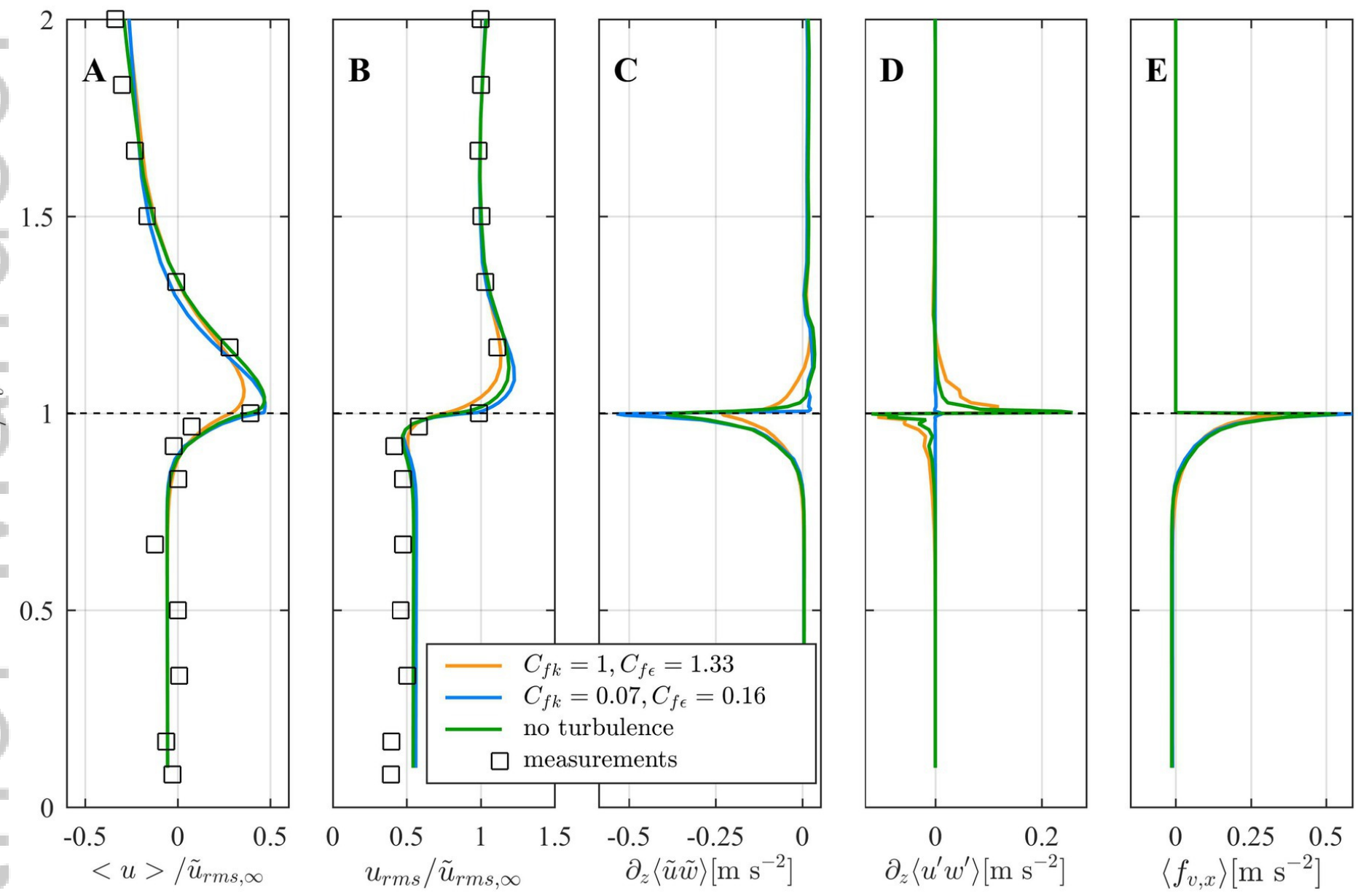

2019JC015935-f11-z-.jpg 


\section{University Library}

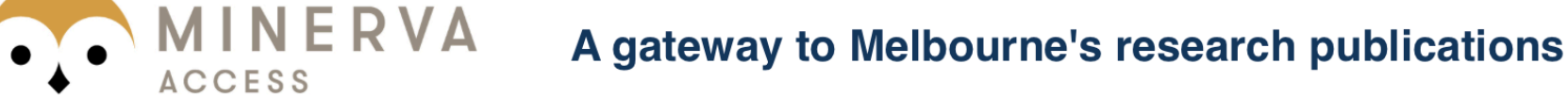

Minerva Access is the Institutional Repository of The University of Melbourne

\section{Author/s:}

van Rooijen, A;Lowe, R;Rijnsdorp, DP;Ghisalberti, M;Jacobsen, NG;McCall, R

Title:

Wave-Driven Mean Flow Dynamics in Submerged Canopies

Date:

2020-03-01

Citation:

van Rooijen, A., Lowe, R., Rijnsdorp, D. P., Ghisalberti, M., Jacobsen, N. G. \& McCall, R. (2020). Wave-Driven Mean Flow Dynamics in Submerged Canopies. JOURNAL OF GEOPHYSICAL RESEARCH-OCEANS, 125 (3), https://doi.org/10.1029/2019JC015935.

Persistent Link:

http://hdl.handle.net/11343/275487 